\title{
CONSTRUCTION OF AXISYMMETRIC STEADY STATES \\ OF AN INVISCID INCOMPRESSIBLE FLUID \\ BY SPATIALLY DISCRETIZED EQUATIONS \\ FOR PSEUDO-ADVECTED VORTICITY
}

TAKAHIRO NISHIYAMA

Received 18 April 2005

An infinite number of generalized solutions to the stationary Euler equations with axisymmetry and prescribed circulation are constructed by applying the finite difference method for spatial variables to an equation of pseudo-advected vorticity. They are proved to be different from exact solutions which are written with trigonometric functions and a Coulomb wave function.

\section{Introduction}

In a domain $\Omega\left(\subset \mathbb{R}^{3}\right)$, the velocity $\mathbf{u}\left(: \Omega \rightarrow \mathbb{R}^{3}\right)$ of a steady-state inviscid incompressible fluid is described by the stationary Euler equations with a boundary condition:

$$
(\mathbf{u} \cdot \nabla) \mathbf{u}=-\nabla p, \quad \nabla \cdot \mathbf{u}=0,\left.\quad \mathbf{u} \cdot \mathbf{n}\right|_{\partial \Omega}=0,
$$

or equivalently,

$$
(\nabla \times \mathbf{u}) \times \mathbf{u}=-\nabla\left(p+\frac{|\mathbf{u}|^{2}}{2}\right), \quad \nabla \cdot \mathbf{u}=0,\left.\quad \mathbf{u} \cdot \mathbf{n}\right|_{\partial \Omega}=0 .
$$

Here, $p(: \Omega \rightarrow \mathbb{R})$ is the pressure, $\partial \Omega$ is the boundary, and $\mathbf{n}$ is the unit outward normal vector on $\partial \Omega$.

In the axisymmetric case, the existence of solutions to (1.2) was discussed as the problem of vortex rings in, for example, $[2,4,5,8]$. Their methods were based on a variational principle for kinetic energy.

By contrast, Vallis et al. [13] proposed a completely different approach to the solvability of (1.2). Assume that the pair $(\mathbf{v}, q): \Omega \times\{t>0\} \rightarrow \mathbb{R}^{3} \times \mathbb{R}$ satisfies the nonstationary system

$$
\mathbf{v}_{t}+\boldsymbol{\omega} \times\left(\mathbf{v}+\alpha \mathbf{v}_{t}\right)=-\nabla\left(q+\frac{|\mathbf{v}|^{2}}{2}\right), \quad \nabla \cdot \mathbf{v}=0,\left.\quad \mathbf{v} \cdot \mathbf{n}\right|_{\partial \Omega}=0
$$


globally in time $t$, where $\boldsymbol{\omega}=\nabla \times \mathbf{v}$, and $\alpha$ is a nonzero constant. They asserted the decay $\mathbf{v}_{t} \rightarrow \mathbf{0}$ and the relaxation of $(\mathbf{v}, q)$ to the above $(\mathbf{u}, p)$ as $t \rightarrow \infty$. For example, in the axisymmetric case (where $\mathbf{v}(\cdot, \cdot, t)$ is a function of the radial and the axial coordinates $r$, $z$ and does not have the azimuthal component), the azimuthal component $\omega$ of $\omega(r, z, t)$ satisfies

$$
\frac{\omega_{t}}{r}+\left(\mathbf{v}+\alpha \mathbf{v}_{t}\right) \cdot \nabla \frac{\omega}{r}=0
$$

It means that the integral $\int_{D} f(\omega / r) r d r d z$ with any smooth function $f$ is conserved in $t$, where $D$ is the cross-section of $\Omega$ in the meridian plane. In addition, from (1.3), we can derive the decay $\int_{D}\left|\mathbf{v}_{t}\right|^{2} r d r d z \rightarrow 0$ as $t \rightarrow \infty$, whether $\alpha<0$ or $\alpha>0$, if $\int_{D}(\omega / r)^{2} r d r d z<$ $\infty$ at $t=0$. This is worthy of remark, because we can obtain an axisymmetric solution to (1.2) which has iso- $(\omega / r)$-lines "topologically accessible" from initially given lines, as was mentioned by Moffatt [7, Section 5].

Some readers may criticize (1.3) saying that it is artificial and unphysical. They should note that in variational approaches to (1.2), all (physical or unphysical) divergence-free fields that deform streamlines or vortex lines are considered in order to obtain energy extrema (see [3, Chapter II, Section 2]). The method of Vallis et al. means that an energy extremum is automatically reached as $t \rightarrow \infty$ if vortex lines are deformed by the divergence-free field $\mathbf{v}+\alpha \mathbf{v}_{t}$.

From a rigorous point of view, the theory of Vallis et al. has not been proved true in its entirety. Indeed, the nonlinearity of $\alpha \boldsymbol{\omega} \times \mathbf{v}_{t}$ seems too strong to obtain the temporally global solvability of (1.3) rigorously.

In order to make use of (1.3) and construct axisymmetric solutions to (1.2) in a rigorous manner, the author in [9] applied the Galerkin method. He approximated (1.3) with $n$ basis functions in $\Omega$ and let $n$ and $t$ go to infinity simultaneously to evade the difficulty of the term $\alpha \boldsymbol{\omega} \times \mathbf{v}_{t}$. (The equality (10) in [9] should be corrected as the inequality $\left\|r^{-1} \nabla \times \mathbf{u}\right\| \leq\left\|r^{-1} \nabla \times \mathbf{v}_{0}\right\|$.)

Nevertheless, a question was left open in [9]. As a set of basis functions, that is, an orthonormal system in a square-integrable space with the weight $r$, the author in [9] used $\left\{\mathbf{w}^{(k)}\right\}_{k \in \mathbb{N}}$ such that each of its elements satisfies (1.2). He could not exclude the possibility of the trivial case in which every constructed solution to (1.2) is written in the form $c \mathbf{w}^{(k)}$ with a constant $c$ and some $k$.

In this paper, we note another system

$$
\mathbf{v}_{t}+\alpha \boldsymbol{\omega} \times P_{\sigma}(\mathbf{v} \times \boldsymbol{\omega})=-\nabla q, \quad \nabla \cdot \mathbf{v}=0,\left.\quad \mathbf{v} \cdot \mathbf{n}\right|_{\partial \Omega}=0,
$$

where $P_{\sigma} \mathbf{f}=\mathbf{f}+\nabla Q$ with $Q$ satisfying $\Delta Q=-\nabla \cdot \mathbf{f}$ and $\left.(\mathbf{f}+\nabla Q) \cdot \mathbf{n}\right|_{\partial \Omega}=0$, and again $\alpha$ is a positive or negative constant. This system was introduced by the author in [11] in the two-dimensional context. It is based on the idea of Vallis et al. Indeed, in the axisymmetric case, it leads to our equation for pseudo-advected vorticity:

$$
\frac{\omega_{t}}{r}+\alpha P_{\sigma}(\mathbf{v} \times \boldsymbol{\omega}) \cdot \nabla \frac{\omega}{r}=0
$$


which has the same property as (1.4). Moreover, (1.5) yields the decay of $\int_{D} \mid P_{\sigma}(\boldsymbol{\omega} \times$ $\mathbf{v})\left.\right|^{2} r d r d z\left(=\int_{D}\left|P_{\sigma}((\mathbf{v} \cdot \nabla) \mathbf{v})\right|^{2} r d r d z\right)$ as $t \rightarrow \infty$ if we assume its temporally global solvability (although it seems difficult to obtain as well as the solvability of (1.3)). Again, some readers may criticize (1.5) for its artificiality. As was mentioned above, it should be taken not as a physical model but as a substitute for variational methods for constructing stationary Euler flows.

The aim of this paper is to approximate (1.6) by the finite difference method for $r, z$ in a cylindrical domain $\Omega$ and prove that it generates an infinite number of generalized solutions to (1.2) which are axisymmetric, periodic in $z$, equipped with prescribed circulation, and different from the above $c \mathbf{w}^{(k)}$. The difficulty of proving the temporally global solvability of (1.6) is evaded by letting the lattice scale $h \rightarrow 0$ and $t \rightarrow \infty$ simultaneously. This is done and a generalized solution to (1.2) is constructed in Section 5 after some preparations in Sections 2, 3 and introducing the approximation of (1.6) in Section 4. For a fundamental theory of the finite difference method, we refer to [6, Chapter VI]. By repeating the process used in Section 5, an infinite number of generalized solutions to (1.2) are generated in Section 6.

In our case, each element of $\left\{\mathbf{w}^{(k)}\right\}_{k \in \mathbb{N}}=\left\{\mathbf{w}^{(m, n)}\right\}_{m \in \mathbb{N}, n \in \mathbb{Z}}$ is concretely written with a trigonometric function and the regular Coulomb wave function of order zero, as is shown in Section 6. It satisfies (1.2). As far as the author knows, no paper introduced this set of exact solutions to (1.2).

An advantage of the finite difference method over the Galerkin method is that we have (5.7), which we mean by the above "prescribed circulation." By virtue of (5.7), we can show that our generalized solutions do not have the form $c \mathbf{w}^{(m, n)}$ (see Theorem 6.1).

In [11], the author discussed the stationary Euler equations in a square domain in $\mathbb{R}^{2}$ by using the finite difference method and proved a theorem analogous to Theorem 5.2. Although our axisymmetric case is more complex, it brings a better result, that is, the construction of an infinite number of generalized solutions in Theorem 6.1. A characteristic of our case is that a small $r$ matches a small lattice scale $h$ and we can prove (3.6), while such an estimate could not be obtained in the planar case in [11].

In the three-dimensional case without axisymmetry, neither (1.3) nor (1.5) seems to be useful, because (1.4) and (1.6) are not valid. We need to use other equations (see $[10,12])$.

\section{Preliminaries}

Let us introduce our notation. We assume that $\Omega$ is a cylindrical domain with a constant radius $a$, that is, $\Omega=\{(r, \theta, z) \mid 0 \leq r<a\}$ and the flow is periodic in $z$. For simplicity, the period is set equal to $a$. The unit vectors in the $r$-, $\theta$-, and $z$-directions in the cylindrical coordinate system are denoted by $\mathbf{e}^{r}, \mathbf{e}^{\theta}$, and $\mathbf{e}^{z}$, respectively.

For $h=a / N$ with a sufficiently large positive integer $N$, we define

$$
\begin{gathered}
\Lambda_{h}=\Lambda_{h}^{r} \times \Lambda_{h}^{z}=\{j \in \mathbb{N} \mid 1 \leq j \leq N-1\} \times\{k \in \mathbb{N} \mid 1 \leq k \leq N\}, \\
\bar{\Lambda}_{h}=\bar{\Lambda}_{h}^{r} \times \Lambda_{h}^{z}=\{j \in \mathbb{Z} \mid 0 \leq j \leq N\} \times\{k \in \mathbb{N} \mid 1 \leq k \leq N\} .
\end{gathered}
$$

The complements of $\Lambda_{h}^{r}$ and $\bar{\Lambda}_{h}^{r}$ in $\mathbb{Z}$ are denoted by $\left(\Lambda_{h}^{r}\right)^{c}$ and $\left(\bar{\Lambda}_{h}^{r}\right)^{c}$, respectively. 
3322 Axisymmetric steady states of an inviscid incompressible fluid

For $\left\{f_{j, k} \in \mathbb{R}\right\}_{(j, k) \in \mathbb{Z}^{2}}$, we define the difference quotients

$$
D_{h, r}^{ \pm} f_{j, k}= \pm \frac{f_{j \pm 1, k}-f_{j, k}}{h}, \quad D_{h, z}^{ \pm} f_{j, k}= \pm \frac{f_{j, k \pm 1}-f_{j, k}}{h} .
$$

These operators $D_{h, r}^{+}, D_{h, r}^{-}, D_{h, z}^{+}$, and $D_{h, z}^{-}$are mutually commutative. For a set of vectors $\left\{\mathbf{f}_{j, k}=f_{j, k}^{r} \mathbf{e}^{r}+f_{j, k}^{z} \mathbf{e}^{z}\right\}_{(j, k) \in \mathbb{Z}^{2}}$, we define

$$
D_{h, s}^{ \pm} \mathbf{f}_{j, k}=\left(D_{h, s}^{ \pm} f_{j, k}^{r}\right) \mathbf{e}^{r}+\left(D_{h, s}^{ \pm} f_{j, k}^{z}\right) \mathbf{e}^{z}
$$

with $s=r$ or $z$. The difference version of the gradient and the divergence operators are defined by

$$
\begin{gathered}
\operatorname{grad}_{h}^{ \pm} f_{j, k}=\left(D_{h, r}^{ \pm} f_{j, k}\right) \mathbf{e}^{r}+\left(D_{h, z}^{ \pm} f_{j, k}\right) \mathbf{e}^{z}, \\
\operatorname{div}_{h}^{ \pm} \mathbf{f}_{j, k}=\frac{1}{(j+1) h} D_{h, r}^{ \pm}\left((j+1) h f_{j, k}^{r}\right)+D_{h, z}^{ \pm} f_{j, k}^{z} .
\end{gathered}
$$

It is easy to verify

$$
\begin{array}{r}
D_{h, r}^{ \pm} f_{j, k}=D_{h, r}^{\mp} f_{j \pm 1, k}, \quad D_{h, z}^{ \pm} f_{j, k}=D_{h, z}^{\mp} f_{j, k \pm 1}, \\
D_{h, r}^{ \pm}\left(f_{j, k} g_{j, k}\right)=\left(D_{h, r}^{ \pm} f_{j, k}\right) g_{j, k}+f_{j \pm 1, k}\left(D_{h, r}^{ \pm} g_{j, k}\right) \\
=\left(D_{h, r}^{ \pm} f_{j, k}\right) g_{j \pm 1, k}+f_{j, k}\left(D_{h, r}^{ \pm} g_{j, k}\right), \\
D_{h, z}^{ \pm}\left(f_{j, k} g_{j, k}\right)=\left(D_{h, z}^{ \pm} f_{j, k}\right) g_{j, k}+f_{j, k \pm 1}\left(D_{h, z}^{ \pm} g_{j, k}\right) \\
=\left(D_{h, z}^{ \pm} f_{j, k}\right) g_{j, k \pm 1}+f_{j, k}\left(D_{h, z}^{ \pm} g_{j, k}\right) .
\end{array}
$$

Furthermore, we have

$$
f_{j, k} D_{h, r}^{ \pm} f_{j, k}=\frac{1}{2}\left(\mp h\left(D_{h, r}^{ \pm} f_{j, k}\right)^{2}+D_{h, r}^{ \pm} f_{j, k}^{2}\right) .
$$

We will often use

$$
\begin{aligned}
& \sum_{j=j_{1}}^{j_{2}}\left(D_{h, r}^{+} f_{j, k}\right) g_{j, k}=-\sum_{j=j_{1}}^{j_{2}} f_{j, k}\left(D_{h, r}^{-} g_{j, k}\right)+\frac{f_{j_{2}+1, k} g_{j_{2}, k}}{h}-\frac{f_{j_{1}, k} g_{j_{1}-1, k}}{h}, \\
& \sum_{k=k_{1}}^{k_{2}}\left(D_{h, z}^{+} f_{j, k}\right) g_{j, k}=-\sum_{k=k_{1}}^{k_{2}} f_{j, k}\left(D_{h, z}^{-} g_{j, k}\right)+\frac{f_{j, k_{2}+1} g_{j, k_{2}}}{h}-\frac{f_{j, k_{1}} g_{j, k_{1}-1}}{h},
\end{aligned}
$$

in particular,

$$
\sum_{(j, k) \in \bar{\Lambda}_{h}}\left(D_{h, s}^{ \pm} f_{j, k}\right) g_{j, k}=-\sum_{(j, k) \in \bar{\Lambda}_{h}} f_{j, k}\left(D_{h, s}^{\mp} g_{j, k}\right)
$$

for $s=r$ if $f_{-1, k}=f_{0, k}=f_{N, k}=f_{N+1, k}=0$ or $f_{-1, k}=f_{N+1, k}=g_{-1, k}=g_{N+1, k}=0$, and for $s=z$ if $f_{j, k}=f_{j, k+N}$ and $g_{j, k}=g_{j, k+N}$. It is also convenient to note the following inequalities. The last one (2.11) is known as the discrete Poincaré inequality. 
Lemma 2.1. If $f_{j_{1}-1, k}=0$, then

$$
\begin{aligned}
& \max _{j_{1} \leq j \leq j_{2}} f_{j, k}^{2} \leq h \delta \sum_{j=j_{1}}^{j_{2}} f_{j, k}^{2}+\frac{h}{\delta} \sum_{j=j_{1}}^{j_{2}}\left(D_{h, r}^{-} f_{j, k}\right)^{2}, \\
& \sum_{j=j_{1}}^{j_{2}} f_{j, k}^{2} \leq 4\left(j_{2}-j_{1}+1\right)^{2} h^{2} \sum_{j=j_{1}}^{j_{2}}\left(D_{h, r}^{-} f_{j, k}\right)^{2}
\end{aligned}
$$

hold, where $\delta>0$ is arbitrary. Particularly, if $f_{0, k}=0$, then

$$
\sum_{j=1}^{N-1} f_{j, k}^{2} \leq 4 a^{2} \sum_{j=1}^{N-1}\left(D_{h, r}^{-} f_{j, k}\right)^{2} .
$$

Proof. Let $l \in \mathbb{Z} \cap\left[j_{1}, j_{2}\right]$. Then, by (2.5) and Schwarz's inequality, we deduce that

$$
\begin{aligned}
f_{l, k}^{2}=h \sum_{j=j_{1}}^{l} D_{h, r}^{-} f_{j, k}^{2} & \leq h \sum_{j=j_{1}}^{j_{2}}\left|D_{h, r}^{-} f_{j, k}\right|\left(\left|f_{j, k}\right|+\left|f_{j-1, k}\right|\right) \\
& \leq 2 h\left(\sum_{j=j_{1}}^{j_{2}} f_{j, k}^{2} \sum_{j=j_{1}}^{j_{2}}\left(D_{h, r}^{-} f_{j, k}\right)^{2}\right)^{1 / 2},
\end{aligned}
$$

which yields (2.9) by Young's inequality and whose summation from $l=j_{1}$ to $j_{2}$ yields (2.10). It is clear that (2.10) with $j_{1}=1$ and $j_{2}=N-1$ leads to (2.11).

Let $\langle\{\cdot, \cdot\}\rangle_{S}$ and $|\{\cdot\}|_{S}$ for sets of scalars $\left\{f_{j, k}\right\},\left\{g_{j, k}\right\}$ or of vectors $\left\{\mathbf{f}_{j, k}\right\},\left\{\mathbf{g}_{j, k}\right\}$ be defined by

$$
\left\langle\left\{f_{j, k}, g_{j, k}\right\}\right\rangle_{S}=h^{3} \sum_{(j, k) \in S}(j+1) f_{j, k} g_{j, k}, \quad\left|\left\{f_{j, k}\right\}\right|_{S}^{2}=\left\langle\left\{f_{j, k}, f_{j, k}\right\}\right\rangle_{S}
$$

or by

$$
\left\langle\left\{\mathbf{f}_{j, k}, \mathbf{g}_{j, k}\right\}\right\rangle_{S}=h^{3} \sum_{(j, k) \in S}(j+1) \mathbf{f}_{j, k} \cdot \mathbf{g}_{j, k}, \quad\left|\left\{\mathbf{f}_{j, k}\right\}\right|_{S}^{2}=\left\langle\left\{\mathbf{f}_{j, k}, \mathbf{f}_{j, k}\right\}\right\rangle_{S} .
$$

In order to discuss the limit $h \rightarrow 0$, it is convenient to use the interpolation operators $A_{h}$ and $B_{h}$ defined by

$$
\begin{aligned}
& A_{h}\left\{f_{j, k}\right\}(r, z)=f_{[r / h],[z / h]}, \\
B_{h}\left\{f_{j, k}\right\}(r, z)= & f_{[r / h],[z / h]}+\left(r-h\left[\frac{r}{h}\right]\right) D_{h, r}^{+} f_{[r / h],[z / h]} \\
& +\left(z-h\left[\frac{z}{h}\right]\right) D_{h, z}^{+} f_{[r / h],[z / h]} \\
& +\left(r-h\left[\frac{r}{h}\right]\right)\left(z-h\left[\frac{z}{h}\right]\right) D_{h, r}^{+} D_{h, z}^{+} f_{[r / h],[z / h]} .
\end{aligned}
$$


3324 Axisymmetric steady states of an inviscid incompressible fluid

Here, $[r / h]$ means the integer in $(r / h-1, r / h]$. These operators correspond to $\tilde{u}_{h}$ and $u_{h}^{\prime}$ in $[6$, Chapter VI]. They are also used for sets of vectors as

$$
A_{h}\left\{f_{j, k}^{r} \mathbf{e}^{r}+f_{j, k}^{z} \mathbf{e}^{z}\right\}=A_{h}\left\{f_{j, k}^{r}\right\} \mathbf{e}^{r}+A_{h}\left\{f_{j, k}^{z}\right\} \mathbf{e}^{z} .
$$

It is useful to note that $B_{h}\left\{f_{j, k}\right\}$ is a continuous and piecewise bilinear function and that the inequalities

$$
\begin{array}{ll}
\left|\frac{\partial}{\partial r} B_{h}\left\{f_{j, k}\right\}(r, z)\right| \leq\left|D_{h, r}^{+} f_{[r / h],[z / h]}\right|+\left|D_{h, r}^{+} f_{[r / h],[z / h]+1}\right| & \text { if } \frac{r}{h} \notin \mathbb{Z}, \\
\left|\frac{\partial}{\partial z} B_{h}\left\{f_{j, k}\right\}(r, z)\right| \leq\left|D_{h, z}^{+} f_{[r / h],[z / h]}\right|+\left|D_{h, z}^{+} f_{[r / h]+1,[z / h]}\right| & \text { if } \frac{z}{h} \notin \mathbb{Z}
\end{array}
$$

are derived from

$$
\begin{aligned}
\left|\frac{\partial}{\partial r} B_{h}\left\{f_{j, k}\right\}(r, z)\right| & =\left|D_{h, r}^{+} f_{[r / h],[z / h]}+\left(z-h\left[\frac{z}{h}\right]\right) D_{h, r}^{+} D_{h, z}^{+} f_{[r / h],[z / h]}\right| \\
& \leq \max \left\{\left|D_{h, r}^{+} f_{[r / h],[z / h]}\right|,\left|D_{h, r}^{+} f_{[r / h],[z / h]+1}\right|\right\}, \\
\left|\frac{\partial}{\partial z} B_{h}\left\{f_{j, k}\right\}(r, z)\right| & =\left|D_{h, z}^{+} f_{[r / h],[z / h]}+\left(r-h\left[\frac{r}{h}\right]\right) D_{h, r}^{+} D_{h, z}^{+} f_{[r / h],[z / h]}\right| \\
& \leq \max \left\{\left|D_{h, z}^{+} f_{[r / h],[z / h]}\right|,\left|D_{h, z}^{+} f_{[r / h]+1,[z / h]}\right|\right\} .
\end{aligned}
$$

Moreover, we have

$$
\begin{aligned}
\mid B_{h}\{ & \left.f_{j, k}\right\}(r, z)-A_{h}\left\{f_{j, k}\right\}(r, z) \mid \\
& \leq h\left|D_{h, r}^{+} f_{[r / h],[z / h]}+\left(z-h\left[\frac{z}{h}\right]\right) D_{h, r}^{+} D_{h, z}^{+} f_{[r / h],[z / h]}\right|+h\left|D_{h, z}^{+} f_{[r / h],[z / h]}\right| \\
& \leq \max \left\{h\left|D_{h, r}^{+} f_{[r / h],[z / h]}\right|, h\left|D_{h, r}^{+} f_{[r / h],[z / h]+1}\right|\right\}+h\left|D_{h, z}^{+} f_{[r / h],[z / h]}\right| \\
& \leq h\left(\left|D_{h, r}^{+} f_{[r / h],[z / h]}\right|+\left|D_{h, r}^{+} f_{[r / h],[z / h]+1}\right|+\left|D_{h, z}^{+} f_{[r / h],[z / h]}\right|\right) .
\end{aligned}
$$

The scalar product $\langle\cdot, \cdot\rangle$ for scalar functions $f(r, z), g(r, z)$ or vector functions $\mathbf{f}(r, z)$, $\mathbf{g}(r, z)$ is defined by

$$
\langle f, g\rangle=\iint_{0}^{a} f(r, z) g(r, z) r d r d z \quad \text { or } \quad\langle\mathbf{f}, \mathbf{g}\rangle=\iint_{0}^{a} \mathbf{f}(r, z) \cdot \mathbf{g}(r, z) r d r d z
$$


The norm $\|\cdot\|$ is defined by $\|f\|=\langle f, f\rangle^{1 / 2}$ or $\|\mathbf{f}\|=\langle\mathbf{f}, \mathbf{f}\rangle^{1 / 2}$. Let $\mathbf{X}_{0}$ and $\mathbf{X}_{1}$ be spaces given by

$$
\begin{gathered}
\mathbf{X}_{0}=\left\{\mathbf{f}=f^{r}(r, z) \mathbf{e}^{r}+f^{z}(r, z) \mathbf{e}^{z},(r, z) \in(0, a) \times \mathbb{R} \mid\|\mathbf{f}\|<\infty,\right. \\
\left.\nabla \cdot \mathbf{f}=0, f^{r}(a, z)=0, \mathbf{f}(r, z)=\mathbf{f}(r, z+a)\right\}
\end{gathered}
$$

with the scalar product $\langle\cdot, \cdot\rangle$ and

$$
\mathbf{X}_{1}=\left\{\mathbf{f} \in \mathbf{X}_{0} \mid\left\|r^{-1} \nabla \times \mathbf{f}\right\|<\infty\right\}
$$

with the scalar product $\left\langle r^{-1} \nabla \times \cdot, r^{-1} \nabla \times \cdot\right\rangle$. We consider the construction of solutions to (1.2) in $\mathbf{X}_{1}$, particularly in a subspace of itself: $\tilde{\mathbf{X}}_{1}=\mathbf{X}_{1} \cap \widetilde{\mathbf{X}}_{0}$. Here, $\tilde{\mathbf{X}}_{0}$ is a subspace of $\mathrm{X}_{0}$ :

$$
\tilde{\mathbf{X}}_{0}=\left\{\mathbf{f} \in \mathbf{X}_{0} \mid \iint_{0}^{a} \mathbf{f} r d r d z=\mathbf{0}\right\}
$$

We also use the Sobolev space of the first order on $(0, a)^{2}$, denoted by $W_{2}^{1}\left((0, a)^{2}\right)$, and the space of vector functions whose components belong to $W_{2}^{1}\left((0, a)^{2}\right)$, denoted by $\mathbf{W}_{2}^{1}\left((0, a)^{2}\right)$.

We sometimes use $\left(\begin{array}{l}f^{r} \\ f^{z}\end{array}\right)$ or $\left(f^{r}, f^{z}\right)$ instead of $f^{r} \mathbf{e}^{r}+f^{z} \mathbf{e}^{z}$.

\section{Difference operator $\Xi_{h}$}

In the axisymmetric case, the relation between the stream function $\phi$ and the vorticity $\omega$ is represented by

$$
\Xi \phi=-r \omega
$$

where

$$
\Xi=r \frac{\partial}{\partial r}\left(\frac{1}{r} \frac{\partial}{\partial r}\right)+\frac{\partial^{2}}{\partial z^{2}}
$$

(see $[2,4,5,8])$.

Let the difference operator $\Xi_{h}$ be defined by

$$
\begin{aligned}
\Xi_{h} f_{j, k} & =(j+1) h D_{h, r}^{+}\left(\frac{1}{(j+1) h} D_{h, r}^{-} f_{j, k}\right)+D_{h, z}^{+} D_{h, z}^{-} f_{j, k} \\
& =D_{h, r}^{+} D_{h, r}^{-} f_{j, k}-\frac{1}{(j+2) h} D_{h, r}^{+} f_{j, k}+D_{h, z}^{+} D_{h, z}^{-} f_{j, k} .
\end{aligned}
$$

It is a difference approximation of $\Xi$.

The following lemmas will be used in Section 5. From now on, we frequently denote positive constants independent of $h$ by $C$ or $C^{\prime}$ without distinction. 
3326 Axisymmetric steady states of an inviscid incompressible fluid

Lemma 3.1. Let $\left\{f_{j, k}\right\}$ be a set such that $f_{j, k}=0$ for all $j \in\left(\Lambda_{h}^{r}\right)^{c}$ and $f_{j, k}=f_{j, k+N}$. Then,

$$
\begin{gathered}
\sum_{(j, k) \in \bar{\Lambda}_{h}}\left|\operatorname{grad}_{h}^{-} f_{j, k}\right|^{2} \leq C \sum_{(j, k) \in \Lambda_{h}}\left(\Xi_{h} f_{j, k}\right)^{2}, \\
\sum_{(j, k) \in \Lambda_{h}}\left(\left|D_{h, r}^{+} \operatorname{grad}_{h}^{-} f_{j, k}\right|^{2}+\left|D_{h, z}^{+} \operatorname{grad}_{h}^{-} f_{j, k}\right|^{2}\right) \leq C \sum_{(j, k) \in \Lambda_{h}}\left(\Xi_{h} f_{j, k}\right)^{2}, \\
\sum_{k=1}^{N}\left|D_{h, r}^{+} \operatorname{grad}_{h}^{-} f_{0, k}\right|^{2} \leq C \sum_{(j, k) \in \Lambda_{h}}\left(\Xi_{h} f_{j, k}\right)^{2}, \\
\sum_{k=1}^{N}\left|D_{h, z}^{+} \operatorname{grad}_{h}^{-} f_{N, k}\right|^{2} \leq C \sum_{(j, k) \in \Lambda_{h}}\left(\Xi_{h} f_{j, k}\right)^{2} .
\end{gathered}
$$

Proof. By (2.6) and (2.8), we have

$$
-\sum_{(j, k) \in \bar{\Lambda}_{h}} f_{j, k} \Xi_{h} f_{j, k}=\sum_{(j, k) \in \bar{\Lambda}_{h}}\left|\operatorname{grad}_{h}^{-} f_{j, k}\right|^{2}+\Sigma_{1},
$$

where

$$
\begin{aligned}
\Sigma_{1} & =\sum_{(j, k) \in \bar{\Lambda}_{h}} \frac{1}{(j+2) h} f_{j, k} D_{h, r}^{+} f_{j, k} \\
& =\sum_{(j, k) \in \bar{\Lambda}_{h}} \frac{1}{2(j+2) h}\left(-h\left(D_{h, r}^{+} f_{j, k}\right)^{2}+D_{h, r}^{+} f_{j, k}^{2}\right) \\
& \geq \sum_{(j, k) \in \bar{\Lambda}_{h}}\left(-\frac{1}{4}\left(D_{h, r}^{+} f_{j, k}\right)^{2}-\left(D_{h, r}^{-} \frac{1}{2(j+2) h}\right) f_{j, k}^{2}\right) \\
& \geq-\frac{1}{4} \sum_{(j, k) \in \bar{\Lambda}_{h}}\left(D_{h, r}^{+} f_{j, k}\right)^{2}=-\frac{1}{4} \sum_{(j, k) \in \bar{\Lambda}_{h}}\left(D_{h, r}^{-} f_{j, k}\right)^{2} .
\end{aligned}
$$

Therefore, using (2.11), we obtain

$$
\begin{aligned}
\frac{3}{4} \sum_{(j, k) \in \bar{\Lambda}_{h}}\left|\operatorname{grad}_{h}^{-} f_{j, k}\right|^{2} & \leq-\sum_{(j, k) \in \bar{\Lambda}_{h}} f_{j, k} \Xi_{h} f_{j, k}=-\sum_{(j, k) \in \Lambda_{h}} f_{j, k} \Xi_{h} f_{j, k} \\
& \leq\left(\sum_{(j, k) \in \Lambda_{h}} f_{j, k}^{2} \sum_{(j, k) \in \Lambda_{h}}\left(\Xi_{h} f_{j, k}\right)^{2}\right)^{1 / 2} \\
& \leq C\left(\sum_{(j, k) \in \bar{\Lambda}_{h}}\left|\operatorname{grad}_{h}^{-} f_{j, k}\right|^{2} \sum_{(j, k) \in \Lambda_{h}}\left(\Xi_{h} f_{j, k}\right)^{2}\right)^{1 / 2}
\end{aligned}
$$

which leads to (3.4). 
Next, we have

$$
\sum_{(j, k) \in \Lambda_{h}}\left(\Xi_{h} f_{j, k}\right)^{2}=\sum_{(j, k) \in \Lambda_{h}}\left(\left(D_{h, r}^{+} D_{h, r}^{-} f_{j, k}\right)^{2}+\frac{\left(D_{h, r}^{+} f_{j, k}\right)^{2}}{(j+2)^{2} h^{2}}+\left(D_{h, z}^{+} D_{h, z}^{-} f_{j, k}\right)^{2}\right)+\Sigma_{2}+\Sigma_{3}+\Sigma_{4},
$$

where

$$
\begin{aligned}
& \Sigma_{2}=-2 \sum_{(j, k) \in \Lambda_{h}} \frac{1}{(j+2) h}\left(D_{h, r}^{+} f_{j, k}\right)\left(D_{h, r}^{+} D_{h, r}^{-} f_{j, k}\right), \\
& \Sigma_{3}=-2 \sum_{(j, k) \in \Lambda_{h}} \frac{1}{(j+2) h}\left(D_{h, r}^{+} f_{j, k}\right)\left(D_{h, z}^{+} D_{h, z}^{-} f_{j, k}\right), \\
& \Sigma_{4}=2 \sum_{(j, k) \in \Lambda_{h}}\left(D_{h, r}^{+} D_{h, r}^{-} f_{j, k}\right)\left(D_{h, z}^{+} D_{h, z}^{-} f_{j, k}\right) .
\end{aligned}
$$

Using (2.6) and (2.7), we derive

$$
\begin{aligned}
\Sigma_{2}= & -\sum_{(j, k) \in \Lambda_{h}} \frac{1}{(j+2) h}\left(h\left(D_{h, r}^{+} D_{h, r}^{-} f_{j, k}\right)^{2}+D_{h, r}^{-}\left(D_{h, r}^{+} f_{j, k}\right)^{2}\right) \\
\geq & \sum_{(j, k) \in \Lambda_{h}}\left(-\frac{1}{3}\left(D_{h, r}^{+} D_{h, r}^{-} f_{j, k}\right)^{2}+\left(D_{h, r}^{+} \frac{1}{(j+2) h}\right)\left(D_{h, r}^{+} f_{j, k}\right)^{2}\right) \\
& +\sum_{k=1}^{N} \frac{1}{h}\left(\frac{\left(D_{h, r}^{+} f_{0, k}\right)^{2}}{3 h}-\frac{\left(D_{h, r}^{+} f_{N-1, k}\right)^{2}}{(N+2) h}\right) .
\end{aligned}
$$

Here, $\left(D_{h, r}^{+} f_{0, k}\right)^{2} /\left(3 h^{2}\right)$ can be replaced by $\left(D_{h, r}^{+} D_{h, r}^{-} f_{0, k}\right)^{2} / 3$, because $D_{h, r}^{+} f_{-1, k}=0$. This is a characteristic of our case, as was mentioned in the penultimate paragraph of Section 1. Furthermore, inequality (2.9) with $f_{j, k}$ replaced by $D_{h, r}^{+} f_{j, k}$ and with $\delta=6 / a, j_{1}=0, j_{2}=$ $N-1$ yields

$$
\begin{aligned}
\sum_{k=1}^{N} \frac{\left(D_{h, r}^{+} f_{N-1, k}\right)^{2}}{(N+2) h^{2}} \leq & \sum_{k=1}^{N} \frac{\left(D_{h, r}^{+} f_{N-1, k}\right)^{2}}{a h} \\
\leq & \sum_{k=1}^{N}\left(\frac{6}{a^{2}} \sum_{j=0}^{N-1}\left(D_{h, r}^{+} f_{j, k}\right)^{2}+\frac{1}{6} \sum_{j=0}^{N-1}\left(D_{h, r}^{+} D_{h, r}^{-} f_{j, k}\right)^{2}\right) \\
\leq & \sum_{(j, k) \in \Lambda_{h}}\left(C\left(\Xi_{h} f_{j, k}\right)^{2}+\frac{1}{6}\left(D_{h, r}^{+} D_{h, r}^{-} f_{j, k}\right)^{2}\right) \\
& +\frac{1}{6} \sum_{k=1}^{N}\left(D_{h, r}^{+} D_{h, r}^{-} f_{0, k}\right)^{2},
\end{aligned}
$$


3328 Axisymmetric steady states of an inviscid incompressible fluid

where we used (3.4). Therefore, we go ahead with the estimation of $\Sigma_{2}$ from below as

$$
\begin{aligned}
\Sigma_{2} \geq & -\sum_{(j, k) \in \Lambda_{h}}\left(\frac{1}{2}\left(D_{h, r}^{+} D_{h, r}^{-} f_{j, k}\right)^{2}+\frac{\left(D_{h, r}^{+} f_{j, k}\right)^{2}}{(j+2)(j+3) h^{2}}+C\left(\Xi_{h} f_{j, k}\right)^{2}\right) \\
& +\frac{1}{6} \sum_{k=1}^{N}\left(D_{h, r}^{+} D_{h, r}^{-} f_{0, k}\right)^{2} .
\end{aligned}
$$

Since $D_{h, z}^{+} D_{h, z}^{-} f_{j, k}=0$ for $j \in\left(\Lambda_{h}^{r}\right)^{c}$, we can change $(j, k) \in \Lambda_{h}$ in $\Sigma_{3}$ and $\Sigma_{4}$ as $(j, k) \in \bar{\Lambda}_{h}$. Then, by (2.6) and (2.8), we have

$$
\begin{aligned}
\Sigma_{3} & =\sum_{(j, k) \in \bar{\Lambda}_{h}} \frac{2}{(j+2) h}\left(D_{h, r}^{+} D_{h, z}^{-} f_{j, k}\right) D_{h, z}^{-} f_{j, k} \\
& =\sum_{(j, k) \in \bar{\Lambda}_{h}} \frac{1}{(j+2) h}\left(-h\left(D_{h, r}^{+} D_{h, z}^{-} f_{j, k}\right)^{2}+D_{h, r}^{+}\left(D_{h, z}^{-} f_{j, k}\right)^{2}\right) \\
& \geq \sum_{(j, k) \in \bar{\Lambda}_{h}}\left(-\frac{1}{2}\left(D_{h, r}^{+} D_{h, z}^{-} f_{j, k}\right)^{2}-\left(D_{h, r}^{-} \frac{1}{(j+2) h}\right)\left(D_{h, z}^{-} f_{j, k}\right)^{2}\right) \\
& \geq-\frac{1}{2} \sum_{(j, k) \in \bar{\Lambda}_{h}}\left(D_{h, r}^{+} D_{h, z}^{-} f_{j, k}\right)^{2}, \\
\Sigma_{4} & =-\sum_{(j, k) \in \bar{\Lambda}_{h}}\left(\left(D_{h, r}^{+} f_{j, k}\right)\left(D_{h, r}^{+} D_{h, z}^{+} D_{h, z}^{-} f_{j, k}\right)+\left(D_{h, r}^{-} f_{j, k}\right)\left(D_{h, r}^{-} D_{h, z}^{+} D_{h, z}^{-} f_{j, k}\right)\right) \\
& =\sum_{(j, k) \in \bar{\Lambda}_{h}}\left(\left(D_{h, r}^{+} D_{h, z}^{-} f_{j, k}\right)^{2}+\left(D_{h, z}^{+} D_{h, r}^{-} f_{j, k}\right)^{2}\right) .
\end{aligned}
$$

Using the above estimates of $\Sigma_{2}, \Sigma_{3}$, and $\Sigma_{4}$, we deduce that

$$
\begin{aligned}
\sum_{(j, k) \in \Lambda_{h}}\left(\Xi_{h} f_{j, k}\right)^{2} \geq & \frac{1}{2} \sum_{(j, k) \in \Lambda_{h}}\left(\left|D_{h, r}^{+} \operatorname{grad}_{h}^{-} f_{j, k}\right|^{2}+\left|D_{h, z}^{+} \operatorname{grad}_{h}^{-} f_{j, k}\right|^{2}\right) \\
& +\sum_{k=1}^{N}\left(\frac{1}{6}\left|D_{h, r}^{+} \operatorname{grad}_{h}^{-} f_{0, k}\right|^{2}+\left(D_{h, z}^{+} D_{h, r}^{-} f_{N, k}\right)^{2}\right) \\
& -C \sum_{(j, k) \in \Lambda_{h}}\left(\Xi_{h} f_{j, k}\right)^{2},
\end{aligned}
$$

which means (3.5), (3.6), and (3.7) (recall that $\left.D_{h, z}^{+} D_{h, z}^{-} f_{N, k}=0\right)$.

Lemma 3.2. Let $\left\{f_{j, k}\right\}$ be as in Lemma 3.1. Then the following inequality is valid:

$$
\sum_{(j, k) \in \bar{\Lambda}_{h}}\left|\frac{\operatorname{grad}_{h}^{-} f_{j, k}}{(j+1) h}\right|^{2} \leq C \sum_{(j, k) \in \Lambda_{h}}\left(\frac{\Xi_{h} f_{j, k}}{(j+1) h}\right)^{2} .
$$


Proof. We define $\Sigma_{5}$ by

$$
\begin{aligned}
\Sigma_{5} & =-\sum_{(j, k) \in \Lambda_{h}} \frac{f_{j, k}}{(j+2) h} \frac{\Xi_{h} f_{j, k}}{(j+1) h}=-\sum_{(j, k) \in \bar{\Lambda}_{h}} \frac{f_{j, k}}{(j+2) h} \frac{\Xi_{h} f_{j, k}}{(j+1) h} \\
& =-\sum_{(j, k) \in \bar{\Lambda}_{h}} \frac{f_{j, k}}{(j+2) h}\left(D_{h, r}^{+}\left(\frac{D_{h, r}^{-} f_{j, k}}{(j+1) h}\right)+\frac{D_{h, z}^{+} D_{h, z}^{-} f_{j, k}}{(j+1) h}\right) \\
& =\sum_{(j, k) \in \bar{\Lambda}_{h}}\left(\left(\frac{D_{h, r}^{-} f_{j, k}}{(j+1) h}\right)^{2}+\left(D_{h, r}^{-} \frac{1}{(j+2) h}\right) \frac{f_{j, k} D_{h, r}^{-} f_{j, k}}{(j+1) h}+\frac{\left(D_{h, z}^{-} f_{j, k}\right)^{2}}{(j+1)(j+2) h^{2}}\right) .
\end{aligned}
$$

The equality (2.6) yields

$$
\left(D_{h, r}^{-} \frac{1}{(j+2) h}\right) \frac{f_{j, k} D_{h, r}^{-} f_{j, k}}{(j+1) h}=-\frac{1}{2(j+2)}\left(\frac{D_{h, r}^{-} f_{j, k}}{(j+1) h}\right)^{2}-\frac{D_{h, r}^{-} f_{j, k}^{2}}{2(j+1)^{2}(j+2) h^{3}} .
$$

Noting that $-\left(D_{h, r}^{-} f_{j, k}\right)^{2} /(j+2) \geq-\left(D_{h, r}^{-} f_{j, k}\right)^{2} / 3$ for $j \in \bar{\Lambda}_{h}^{r}$ (because $D_{h, r}^{-} f_{0, k}=0$ ), we have

$$
-\frac{1}{6} \sum_{(j, k) \in \bar{\Lambda}_{h}}\left(\frac{D_{h, r}^{-} f_{j, k}}{(j+1) h}\right)^{2}-\frac{\Sigma_{6}}{2} \leq \sum_{(j, k) \in \bar{\Lambda}_{h}}\left(D_{h, r}^{-} \frac{1}{(j+2) h}\right) \frac{f_{j, k} D_{h, r}^{-} f_{j, k}}{(j+1) h} \leq-\frac{\Sigma_{6}}{2},
$$

where

$$
\begin{aligned}
\Sigma_{6} & =\sum_{(j, k) \in \bar{\Lambda}_{h}} \frac{D_{h, r}^{-} f_{j, k}^{2}}{(j+1)^{2}(j+2) h^{3}} \\
& =-\sum_{(j, k) \in \bar{\Lambda}_{h}} f_{j, k}^{2}\left(D_{h, r}^{+} \frac{1}{(j+1)^{2}(j+2) h^{3}}\right) \\
& =\sum_{(j, k) \in \Lambda_{h}} \frac{(3 j+5) f_{j, k}^{2}}{(j+1)^{2}(j+2)^{2}(j+3) h^{4}} .
\end{aligned}
$$

By (3.21), we estimate $\Sigma_{5}$ from below as

$$
\Sigma_{5} \geq \sum_{(j, k) \in \bar{\Lambda}_{h}}\left(\frac{5}{6}\left(\frac{D_{h, r}^{-} f_{j, k}}{(j+1) h}\right)^{2}+\frac{2}{3}\left(\frac{D_{h, z}^{-} f_{j, k}}{(j+1) h}\right)^{2}\right)-\frac{\Sigma_{6}}{2} .
$$


3330 Axisymmetric steady states of an inviscid incompressible fluid

Next, let us estimate $\Sigma_{5}$ from above. By Schwarz's and Young's inequalities, (2.11) and (3.21), we deduce that

$$
\begin{aligned}
\Sigma_{5} \leq & \frac{19}{24} \sum_{(j, k) \in \Lambda_{h}}\left(D_{h, r}^{-} \frac{f_{j, k}}{(j+2) h}\right)^{2}+C \sum_{(j, k) \in \Lambda_{h}}\left(\frac{\Xi_{h} f_{j, k}}{(j+1) h}\right)^{2} \\
= & \frac{19}{24} \sum_{(j, k) \in \Lambda_{h}}\left(\left(\frac{D_{h, r}^{-} f_{j, k}}{(j+1) h}\right)^{2}+2\left(D_{h, r}^{-} \frac{1}{(j+2) h}\right) \frac{f_{j, k} D_{h, r}^{-} f_{j, k}}{(j+1) h}\right. \\
& \left.+\left(D_{h, r}^{-} \frac{1}{(j+2) h}\right)^{2} f_{j, k}^{2}\right)+C \sum_{(j, k) \in \Lambda_{h}}\left(\frac{\Xi_{h} f_{j, k}}{(j+1) h}\right)^{2} \\
\leq & \frac{19}{24} \sum_{(j, k) \in \Lambda_{h}}\left(\left(\frac{D_{h, r}^{-} f_{j, k}}{(j+1) h}\right)^{2}+\frac{f_{j, k}^{2}}{(j+1)^{2}(j+2)^{2} h^{4}}\right) \\
& -\frac{19}{24} \sum_{6}+C \sum_{(j, k) \in \Lambda_{h}}\left(\frac{\Xi_{h} f_{j, k}}{(j+1) h}\right)^{2} .
\end{aligned}
$$

This estimate and (3.23) yield

$$
\begin{aligned}
\frac{1}{24} \sum_{(j, k) \in \bar{\Lambda}_{h}}\left|\frac{\operatorname{grad}_{h}^{-} f_{j, k}}{(j+1) h}\right|^{2} & \leq \sum_{(j, k) \in \Lambda_{h}}\left(\frac{(19 / 24) f_{j, k}^{2}}{(j+1)^{2}(j+2)^{2} h^{4}}+C\left(\frac{\Xi_{h} f_{j, k}}{(j+1) h}\right)^{2}\right)-\frac{7}{24} \Sigma_{6} \\
& \leq \sum_{(j, k) \in \Lambda_{h}}\left(\frac{(11-j) f_{j, k}^{2}}{12(j+1)^{2}(j+2)^{2}(j+3) h^{4}}+C\left(\frac{\Xi_{h} f_{j, k}}{(j+1) h}\right)^{2}\right) \\
& \leq C^{\prime} \sum_{j=1}^{10} \sum_{k=1}^{N} \frac{f_{j, k}^{2}}{h^{4}}+C \sum_{(j, k) \in \Lambda_{h}}\left(\frac{\Xi_{h} f_{j, k}}{(j+1) h}\right)^{2} .
\end{aligned}
$$

Since

$$
\sum_{j=1}^{10} f_{j, k}^{2} \leq C h^{2} \sum_{j=1}^{10}\left(D_{h, r}^{-} f_{j, k}\right)^{2} \leq C^{\prime} h^{4} \sum_{j=1}^{10}\left(D_{h, r}^{-} D_{h, r}^{-} f_{j, k}\right)^{2}
$$

follows from (2.10), we have

$$
\sum_{j=1}^{10} \sum_{k=1}^{N} \frac{f_{j, k}^{2}}{h^{4}} \leq C \sum_{j=0}^{9} \sum_{k=1}^{N}\left(D_{h, r}^{+} D_{h, r}^{-} f_{j, k}\right)^{2} \leq C^{\prime} \sum_{(j, k) \in \Lambda_{h}}\left(\Xi_{h} f_{j, k}\right)^{2} \leq C^{\prime} a^{2} \sum_{(j, k) \in \Lambda_{h}}\left(\frac{\Xi_{h} f_{j, k}}{(j+1) h}\right)^{2}
$$

by using (3.5) and (3.6). Hence, (3.18) holds. 


\section{Spatially discretized equations for pseudo-advected vorticity}

First, we introduce the linear system for $\left\{\phi_{j, k}\right\}_{(j, k) \in \mathbb{Z}^{2}}$ with $\left\{\zeta_{j, k}\right\}_{(j, k) \in \Lambda_{h}}$ given that

$$
\begin{gathered}
\Xi_{h} \phi_{j, k}=-(j+1)^{2} h^{2} \zeta_{j, k} \quad \text { for }(j, k) \in \Lambda_{h}, \\
\left.\phi_{j, k}\right|_{j \in\left(\Lambda_{h}^{r}\right)^{c}}=0, \quad \phi_{j, k}=\phi_{j, k+N} .
\end{gathered}
$$

It is uniquely solvable. Indeed, if $\zeta_{j, k}=0$ is assumed for all $(j, k)$, then $\phi_{j, k}=0$ is derived from (3.4) with (2.11). Therefore, we can represent $\phi_{j, k}$ in $\Lambda_{h}$ as

$$
\phi_{j, k}=\sum_{\left(j^{\prime}, k^{\prime}\right) \in \Lambda_{h}} L_{j, k}^{j^{\prime}, k^{\prime}} \zeta_{j^{\prime}, k^{\prime}}
$$

with constants $\left\{L_{j, k}^{j^{\prime}, k^{\prime}}\right\}(j, k),\left(j^{\prime}, k^{\prime}\right) \in \Lambda_{h}$.

Next, suppose that $\mathbf{f}_{j, k}=\left(f_{j, k}^{r}, f_{j, k}^{z}\right)$ is given for every $(j, k) \in \Lambda_{h}^{r} \times \mathbb{Z}$ so that $\mathbf{f}_{j, k}=\mathbf{f}_{j, k+N}$, and $\left\{f_{0, k}^{r} \mid f_{0, k}^{r}=f_{0, k+N}^{r}\right\}_{k \in \mathbb{Z}}$ is also given. We define the operator $P_{\sigma, h}$ by

$$
P_{\sigma, h} \mathbf{f}_{j, k}=\mathbf{f}_{j, k}+\operatorname{grad}_{h}^{+} Q_{j, k},
$$

where $Q_{j, k}$ is determined by

$$
\begin{gathered}
\operatorname{div}_{h}^{-} \operatorname{grad}_{h}^{+} Q_{j, k}=-\operatorname{div}_{h}^{-} \mathbf{f}_{j, k} \quad \text { for }(j, k) \in \Lambda_{h}, \\
D_{h, r}^{+} Q_{0, k}+f_{0, k}^{r}=0, \quad D_{h, r}^{+} Q_{N-1, k}+f_{N-1, k}^{r}=0, \\
Q_{j, k}=Q_{j, k+N} .
\end{gathered}
$$

It is easily verified that $\operatorname{grad}_{h}^{+} Q_{j, k}$ is uniquely determined for given $\left\{\mathbf{f}_{j, k}\right\}$ and $\left\{f_{0, k}^{r}\right\}$. Indeed, if $\left.\mathbf{f}_{j, k}\right|_{(j, k) \in \Lambda_{h}^{r} \times \mathbb{Z}}=\mathbf{0}$ with $\left.f_{0, k}^{r}\right|_{k \in \mathbb{Z}}=0$ is assumed, then, by using (2.7), we have

$$
0=\left\langle\left\{\operatorname{div}_{h}^{-} \operatorname{grad}_{h}^{+} Q_{j, k}, Q_{j, k}\right\}\right\rangle_{\Lambda_{h}}=-\left|\left\{\operatorname{grad}_{h}^{+} Q_{j, k}\right\}\right|_{\Lambda_{h}}^{2},
$$

which means that $Q_{j, k}=$ const. in $\bar{\Lambda}_{h}^{r} \times \mathbb{Z}$. It is clear that $\operatorname{div}_{h}^{-} P_{\sigma, h} \mathbf{f}_{j, k}=0$ in $\Lambda_{h}$ and $\left(P_{\sigma, h} \mathbf{f}_{j, k}\right) \cdot \mathbf{e}^{r}=0$ for $j=0, N-1$. In addition,

$$
\left\langle\left\{\mathbf{f}_{j, k}, P_{\sigma, h} \mathbf{g}_{j, k}\right\}\right\rangle_{\Lambda_{h}}=\left\langle\left\{P_{\sigma, h} \mathbf{f}_{j, k}, P_{\sigma, h} \mathbf{g}_{j, k}\right\}\right\rangle_{\Lambda_{h}},
$$

because $\left\langle\left\{\operatorname{grad}_{h}^{+} Q_{j, k}, P_{\sigma, h} \mathbf{g}_{j, k}\right\}\right\rangle_{\Lambda_{h}}=-\left\langle\left\{Q_{j, k}, \operatorname{div}_{h}^{-} P_{\sigma, h} \mathbf{g}_{j, k}\right\}\right\rangle_{\Lambda_{h}}=0$.

As an approximation of (1.6) by the finite difference method for $r$ and $z$, we present the system of ordinary differential equations for $\zeta_{j, k}(t)\left((j, k) \in \Lambda_{h}\right)$ :

$$
\frac{d \zeta_{j, k}}{d t}+\frac{\alpha}{2} \mathbf{V}_{j, k} \cdot \operatorname{grad}_{h}^{+} \zeta_{j, k}+\frac{\alpha}{2}\left(\begin{array}{c}
\frac{j}{j+1} V_{j-1, k}^{r} \\
V_{j, k-1}^{z}
\end{array}\right) \cdot \operatorname{grad}_{h}^{-} \zeta_{j, k}=0
$$


3332 Axisymmetric steady states of an inviscid incompressible fluid

with $\left.\zeta_{j, k}\right|_{j \in\left(\Lambda_{h}^{r}\right)^{c}}=0$ and $\zeta_{j, k}=\zeta_{j, k+N}$. Here $\alpha$ is a fixed positive or negative number, and

$$
\mathbf{V}_{j, k}=\left(\begin{array}{c}
V_{j, k}^{r} \\
V_{j, k}^{z}
\end{array}\right)=-\frac{1}{2} P_{\sigma, h}\left(\begin{array}{c}
\left(\zeta_{j, k}+\zeta_{j+1, k}\right) D_{h, r}^{+} \phi_{j, k} \\
\left(\zeta_{j, k}+\zeta_{j, k+1}\right) D_{h, z}^{+} \phi_{j, k}
\end{array}\right)
$$

with $\phi_{j, k}$ given by (4.3) for $(j, k) \in \Lambda_{h}$ and by (4.2) for the others.

It should be noted that (4.1) is not always valid for $j=0$ or $N$. The sets $\left\{\zeta_{j, k}\right\}$ and $\left\{\phi_{j, k}\right\}$ correspond to $\omega / r$ in (1.6) and $\phi$ in (3.1), respectively. As a result of the first condition of (4.2), the mean velocity in $(0, a)^{2}$ of our flows in Theorem 5.2 is equal to zero, while the case with $\left.\phi_{j, k}\right|_{j \leq 0}=0$ and $\left.\phi_{j, k}\right|_{j \geq N}=$ const. $\neq 0$ is open.

Clearly, the system (4.8) is uniquely solvable at least locally in time if we give the initial data

$$
\zeta_{j, k}(0)=\zeta_{0, j, k}
$$

\section{Construction of solutions to the stationary Euler equations}

Let us define a generalized solution to (1.2).

Definition 5.1. If $\mathbf{u} \in \mathbf{X}_{1}$ satisfies

$$
\langle(\nabla \times \mathbf{u}) \times \mathbf{u}, \mathbf{f}\rangle=0
$$

for any $\mathbf{f} \in \mathbf{X}_{1}$, then $\mathbf{u}$ is said to be an axisymmetric generalized solution to (1.2).

If this generalized solution belongs to the $C^{1}$-class, then it is a classical solution to (1.2), according to the well-known orthogonality of the divergence-free and the gradient fields.

Let

$$
\mathbf{v}_{j, k}^{(h)}=\left(-\frac{1}{(j+1) h} D_{h, z}^{-} \phi_{j, k}, \frac{1}{(j+1) h} D_{h, r}^{-} \phi_{j, k}\right) .
$$

Then we have the following theorem.

Theorem 5.2. Assume that $\zeta_{0}$ is an arbitrary function on $(0, a) \times \mathbb{R}$ which satisfies $\zeta_{0}(r, z)=$ $\zeta_{0}(r, z+a)$ and $\left\|\zeta_{0}\right\|<\infty$. Let $\left\{\zeta_{0, j, k}\right\}_{(j, k) \in \mathbb{Z}^{2}}$ be a set such that $\zeta_{0, j, k}=0$ for $j \in\left(\Lambda_{h}^{r}\right)^{c}, \zeta_{0, j, k}=$ $\zeta_{0, j, k+N}$, and $A_{h}\left\{(j+1)^{1 / 2} h^{1 / 2} \zeta_{0, j, k}\right\}$ converges to $r^{1 / 2} \zeta_{0}$ strongly in $L^{2}\left((0, a)^{2}\right)$ as $h \rightarrow 0$. Then (4.8) with (4.10) is uniquely solvable globally in time. There exist sequences

$$
\left\{h_{n} \mid h_{n}>h_{n+1}>0, \lim _{n \rightarrow \infty} h_{n}=0\right\}, \quad\left\{t_{n} \mid 0<t_{n}<t_{n+1}, \lim _{n \rightarrow \infty} t_{n}=\infty\right\}
$$

$(n=1,2,3, \ldots)$ such that

$$
B_{h_{n}}\left\{(j+1) h_{n} \mathbf{v}_{j, k}^{\left(h_{n}\right)}\left(t_{n}\right)\right\} \longrightarrow r \mathbf{u}\left\{\begin{array}{l}
\text { strongly in } \mathbf{L}^{2}\left((0, a)^{2}\right), \\
\text { weakly in } \mathbf{W}_{2}^{1}\left((0, a)^{2}\right),
\end{array}\right.
$$


as $n \rightarrow \infty$, where $\mathbf{u} \in \tilde{\mathbf{X}}_{1}$ is an axisymmetric generalized solution to (1.2). Furthermore,

$$
A_{h_{n}}\left\{(j+1)^{1 / 2} h_{n}^{1 / 2} \zeta_{j, k}\left(t_{n}\right)\right\} \longrightarrow \frac{(\nabla \times \mathbf{u}) \cdot \mathbf{e}^{\theta}}{r^{1 / 2}} \text { weakly in } L^{2}\left((0, a)^{2}\right)
$$

and

$$
\begin{gathered}
\left\|r^{-1} \nabla \times \mathbf{u}\right\| \leq\left\|\zeta_{0}\right\| \\
\iint_{0}^{a}(\nabla \times \mathbf{u}) \cdot \mathbf{e}^{\theta} d r d z=\iint_{0}^{a} \zeta_{0} r d r d z .
\end{gathered}
$$

Proof. First, it should be noted that there exists a set $\left\{\zeta_{0, j, k}\right\}$ satisfying the above convergence of $A_{h}\left\{(j+1)^{1 / 2} h^{1 / 2} \zeta_{0, j, k}\right\}$. Indeed, it is satisfied, for example, if

$$
\zeta_{0, j, k}=\frac{1}{(j+1)^{1 / 2} h^{5 / 2}} \int_{k h}^{(k+1) h} d z \int_{j h}^{(j+1) h} \zeta_{0} r^{1 / 2} d r \quad \text { for }(j, k) \in \Lambda_{h}
$$

(see [6, Chapter VI, Lemma 4.1]).

Let $\left\{\xi_{j, k}\right\}$ and $\left\{\eta_{j, k}\right\}$ be sets such that $\xi_{j, k}=\xi_{j, k+N}$ and $\eta_{j, k}=\eta_{j, k+N}$. Then we can verify that

$$
\begin{aligned}
& \left\langle\left\{\xi_{j, k},\left(\begin{array}{c}
\frac{j}{j+1} V_{j-1, k}^{r} \\
V_{j, k-1}^{z}
\end{array}\right) \cdot \operatorname{grad}_{h}^{-} \eta_{j, k}\right\}\right\rangle_{\Lambda_{h}} \\
& =-\sum_{(j, k) \in \Lambda_{h}}\left(D_{h, r}^{+}\left(j h^{3} \xi_{j, k} V_{j-1, k}^{r}\right)+D_{h, z}^{+}\left((j+1) h^{3} \xi_{j, k} V_{j, k-1}^{z}\right)\right) \eta_{j, k} \\
& =-\sum_{(j, k) \in \Lambda_{h}}(j+1) h^{3}\left(\mathbf{V}_{j, k} \cdot \operatorname{grad}_{h}^{+} \xi_{j, k}+\xi_{j, k} \operatorname{div}_{h}^{-} \mathbf{V}_{j, k}\right) \eta_{j, k} \\
& =-\left\langle\left\{\eta_{j, k}, \mathbf{V}_{j, k} \cdot \operatorname{grad}_{h}^{+} \xi_{j, k}\right\}\right\rangle_{\Lambda_{h}}
\end{aligned}
$$

by (2.5), (2.7), the equalities $V_{0, k}^{r}=V_{N-1, k}^{r}=0$, and $\operatorname{div}_{h}^{-} \mathbf{V}_{j, k}=0$. Summing up the products of (4.8) and $(j+1) h^{3} \zeta_{j, k}$ for $(j, k) \in \Lambda_{h}$ and using (5.9) with $\xi_{j, k}=\eta_{j, k}=\zeta_{j, k}$, we derive $(d / d t)\left|\left\{\zeta_{j, k}\right\}\right|_{\Lambda_{h}}^{2}=0$, which leads to

$$
\left|\left\{\zeta_{j, k}\right\}\right|_{\Lambda_{h}}^{2}=\left|\left\{\zeta_{0, j, k}\right\}\right|_{\Lambda_{h}}^{2}=\iint_{0}^{a}\left(A_{h}\left\{(j+1)^{1 / 2} h^{1 / 2} \zeta_{0, j, k}\right\}\right)^{2} d r d z \leq C \mid\left\|\zeta_{0}\right\|^{2}
$$


3334 Axisymmetric steady states of an inviscid incompressible fluid

This implies that (4.8) is solvable globally in time. In addition, applying (2.11), (3.4)(3.7), (3.18), and (4.1), we deduce that

$$
\begin{aligned}
& h^{2} \sum_{(j, k) \in \bar{\Lambda}_{h}}\left(\phi_{j, k}^{2}+\left|\operatorname{grad}_{h}^{-} \phi_{j, k}\right|^{2}\right) \\
& \quad+h^{2} \sum_{j=0}^{N-1} \sum_{k=1}^{N}\left|D_{h, r}^{+} \operatorname{grad}_{h}^{-} \phi_{j, k}\right|^{2}+h^{2} \sum_{j=1}^{N} \sum_{k=1}^{N}\left|D_{h, z}^{+} \operatorname{grad}_{h}^{-} \phi_{j, k}\right|^{2} \\
& \leq C h^{2} \sum_{(j, k) \in \Lambda_{h}}(j+1)^{4} h^{4} \zeta_{j, k}^{2} \leq C a^{3}\left|\left\{\zeta_{j, k}\right\}\right|_{\Lambda_{h}}^{2} \leq C^{\prime}\left\|\zeta_{0}\right\|^{2}, \\
& h^{2} \sum_{(j, k) \in \bar{\Lambda}_{h}}\left|\mathbf{v}_{j, k}^{(h)}\right|^{2} \leq C h^{2} \sum_{(j, k) \in \Lambda_{h}}(j+1)^{2} h^{2} \zeta_{j, k}^{2} \leq C a\left|\left\{\zeta_{j, k}\right\}\right|_{\Lambda_{h}}^{2} \leq C^{\prime}|| \zeta_{0} \|^{2} .
\end{aligned}
$$

Using

$$
\begin{aligned}
\frac{1}{2} \frac{d}{d t}\left|\left\{\mathbf{v}_{j, k}^{(h)}\right\}\right|_{\bar{\Lambda}_{h}}^{2} & =h^{2} \sum_{(j, k) \in \bar{\Lambda}_{h}} \frac{\operatorname{grad}_{h}^{-} \phi_{j, k}}{(j+1) h} \cdot \operatorname{grad}_{h}^{-}\left(\frac{d}{d t} \phi_{j, k}\right) \\
& =-h^{2} \sum_{(j, k) \in \bar{\Lambda}_{h}} \frac{\phi_{j, k}}{(j+1) h} \Xi_{h}\left(\frac{d}{d t} \phi_{j, k}\right) \\
& =\left\langle\left\{\phi_{j, k}, \frac{d}{d t} \zeta_{j, k}\right\}\right\rangle_{\Lambda_{h}},
\end{aligned}
$$

$V_{0, k}^{r}=V_{N-1, k}^{r}=0$, the periodicity of $\mathbf{V}_{j, k}$ in $k,(4.7)$, and (5.9), we have

$$
\begin{aligned}
\frac{1}{2} \frac{d}{d t} \mid & \left.\left\{\mathbf{v}_{j, k}^{(h)}\right\}\right|_{\bar{\Lambda}_{h}} ^{2} \\
& =-\frac{\alpha}{2}\left\langle\left\{\phi_{j, k}, \mathbf{V}_{j, k} \cdot \operatorname{grad}_{h}^{+} \zeta_{j, k}+\left(\begin{array}{c}
\frac{j}{j+1} V_{j-1, k}^{r} \\
V_{j, k-1}^{z}
\end{array}\right) \cdot \operatorname{grad}_{h}^{-} \zeta_{j, k}\right\}\right\rangle_{\Lambda_{h}} \\
& =\frac{\alpha h^{2}}{2} \sum_{(j, k) \in \Lambda_{h}} \zeta_{j, k}\left((j+1) h \mathbf{V}_{j, k} \cdot \operatorname{grad}_{h}^{+} \phi_{j, k}+\left(\begin{array}{c}
j h V_{j-1, k}^{r} \\
(j+1) h V_{j, k-1}^{z}
\end{array}\right) \cdot \operatorname{grad}_{h}^{-} \phi_{j, k}\right) \\
& =\frac{\alpha h^{2}}{2} \sum_{(j, k) \in \Lambda_{h}}(j+1) h \mathbf{V}_{j, k} \cdot\left(\begin{array}{c}
\left(\zeta_{j, k}+\zeta_{j+1, k}\right) D_{h, r}^{+} \phi_{j, k} \\
\left(\zeta_{j, k}+\zeta_{j, k+1}\right) D_{h, z}^{+} \phi_{j, k}
\end{array}\right) \\
& =-\alpha\left|\left\{\mathbf{V}_{j, k}\right\}\right|_{\Lambda_{h}}^{2} .
\end{aligned}
$$


From this and (5.12), we derive

$$
\begin{aligned}
\int_{0}^{t}\left|\left\{\mathbf{V}_{j, k}\right\}\right|_{\Lambda_{h}}^{2} d t & =-\frac{1}{2 \alpha}\left(\left|\left\{\mathbf{v}_{j, k}^{(h)}(t)\right\}\right|_{\bar{\Lambda}_{h}}^{2}-\left|\left\{\mathbf{v}_{0, j, k}^{(h)}\right\}\right|_{\bar{\Lambda}_{h}}^{2}\right) \\
& \leq \frac{a}{2|\alpha|} \sup _{t^{\prime} \geq 0} h^{2} \sum_{(j, k) \in \bar{\Lambda}_{h}}\left|\mathbf{v}_{j, k}^{(h)}\left(t^{\prime}\right)\right|^{2} \leq C_{1}\left\|\zeta_{0}\right\|^{2},
\end{aligned}
$$

where $\mathbf{v}_{0, j, k}^{(h)}=\left.\mathbf{v}_{j, k}^{(h)}\right|_{t=0}$ and $C_{1}$ is a positive constant independent of $h$. Since (5.15) means the integrability of $\left|\left\{\mathbf{V}_{j, k}(t)\right\}\right|_{\Lambda_{h}}^{2}$ over $(0, \infty)$, there exists a sequence

$$
\left\{t_{n}^{(h)} \mid 2^{n-1}<t_{n}^{(h)}<2^{n}\right\} \quad(n=1,2,3, \ldots)
$$

for each $h$ such that

$$
\left|\left\{\mathbf{V}_{j, k}\left(t_{n}^{(h)}\right)\right\}\right|_{\Lambda_{h}}^{2} \leq \frac{C_{1}\left\|\zeta_{0}\right\|^{2}}{2^{n-1}}
$$

Indeed, if the nonexistence of this $\left\{t_{n}^{(h)}\right\}$ is supposed, then we have

$$
\int_{2^{n-1}}^{2^{n}}\left|\left\{\mathbf{V}_{j, k}\right\}\right|_{\Lambda_{h}}^{2} d t>\left(2^{n}-2^{n-1}\right) \frac{C_{1}\left\|\zeta_{0}\right\|^{2}}{2^{n-1}}=C_{1}\left\|\zeta_{0}\right\|^{2}
$$

which contradicts (5.15).

Noting (5.11), the equality $\sum_{(j, k) \in \bar{\Lambda}_{h}}\left|\operatorname{grad}_{h}^{+} \phi_{j, k}\right|^{2}=\sum_{(j, k) \in \bar{\Lambda}_{h}}\left|\operatorname{grad}_{h}^{-} \phi_{j, k}\right|^{2}$, and [6, Chapter VI, Theorem 3.1], we have the existence of a sequence

$$
\left\{h_{n} \mid h_{n}>h_{n+1}>0, \lim _{n \rightarrow \infty} h_{n}=0\right\} \quad(n=1,2,3, \ldots)
$$

such that $B_{h_{n}}\left\{\phi_{j, k}\left(t_{n}\right)\right\}$ with $t_{n}=t_{n}^{\left(h_{n}\right)}$ converges to a function $\psi \in W_{2}^{1}\left((0, a)^{2}\right)$ strongly in $L^{2}\left((0, a)^{2}\right)$ and weakly in $W_{2}^{1}\left((0, a)^{2}\right)$ as $n \rightarrow \infty$. Here, $\left.\psi\right|_{r=0, a}=0$ and $\psi(r, z)=\psi(r, z+a)$ hold. By virtue of [6, Chapter VI, Lemma 3.1], we derive that

$$
A_{h_{n}}\left\{\operatorname{grad}_{h_{n}}^{+} \phi_{j, k}\left(t_{n}\right)\right\} \longrightarrow \nabla \psi \quad \text { weakly in } \mathbf{L}^{2}\left((0, a)^{2}\right)
$$

In the same way as $B_{h_{n}}\left\{\phi_{j, k}\left(t_{n}\right)\right\}$, both of $B_{h_{n}}\left\{\phi_{j-1, k}\left(t_{n}\right)\right\}$ and $B_{h_{n}}\left\{\phi_{j, k-1}\left(t_{n}\right)\right\}$ also converge to $\psi$ strongly in $L^{2}\left((0, a)^{2}\right)$ and weakly in $W_{2}^{1}\left((0, a)^{2}\right)$. Indeed, the fact that the limit of $B_{h_{n}}\left\{\phi_{j-1, k}\left(t_{n}\right)\right\}$ is $\psi$ follows from

$$
\begin{gathered}
\iint_{0}^{a}\left(B_{h_{n}}\left\{\phi_{j-1, k}\left(t_{n}\right)\right\}(r, z)-\psi\left(r-h_{n}, z\right)\right)^{2} d r d z \longrightarrow 0, \\
\iint_{0}^{a}\left(\psi\left(r-h_{n}, z\right)-\psi(r, z)\right)^{2} d r d z \longrightarrow 0 \quad \text { (the Lebesgue theorem), }
\end{gathered}
$$


3336 Axisymmetric steady states of an inviscid incompressible fluid

where we set $\psi=0$ for $r<0$. Then,

$$
\left(\begin{array}{l}
\frac{\partial}{\partial r} B_{h_{n}}\left\{\phi_{j-1, k}\left(t_{n}\right)\right\} \\
\frac{\partial}{\partial z} B_{h_{n}}\left\{\phi_{j, k-1}\left(t_{n}\right)\right\}
\end{array}\right) \longrightarrow \nabla \psi \quad \text { weakly in } \mathbf{L}^{2}\left((0, a)^{2}\right)
$$

According to [6, Chapter VI, Lemma 3.1], this implies that

$$
B_{h_{n}}\left\{\operatorname{grad}_{h_{n}}^{-} \phi_{j, k}\left(t_{n}\right)\right\} \longrightarrow \nabla \psi \quad \text { weakly in } \mathbf{L}^{2}\left((0, a)^{2}\right)
$$

Remark that

$$
\begin{aligned}
& \iint_{0}^{a}\left(\left|\frac{\partial}{\partial r} B_{h}\left\{\operatorname{grad}_{h}^{-} \phi_{j, k}\right\}\right|^{2}+\left|\frac{\partial}{\partial z} B_{h}\left\{\operatorname{grad}_{h}^{-} \phi_{j, k}\right\}\right|^{2}\right) d r d z \\
& \quad \leq 4 h^{2} \sum_{j=0}^{N-1} \sum_{k=1}^{N}\left|D_{h, r}^{+} \operatorname{grad}_{h}^{-} \phi_{j, k}\right|^{2}+4 h^{2} \sum_{j=0}^{N} \sum_{k=1}^{N}\left|D_{h, z}^{+} \operatorname{grad}_{h}^{-} \phi_{j, k}\right|^{2} \leq C\left\|\zeta_{0}\right\|^{2}
\end{aligned}
$$

follows from (2.17), (5.11), and $\left|D_{h, z}^{+} \operatorname{grad}_{h}^{-} \phi_{0, k}\right|=0$. Then, by Rellich's theorem, we deduce that $\nabla \psi$ belongs to $\mathbf{W}_{2}^{1}\left((0, a)^{2}\right)$ and $(5.23)$ is improved as

$$
B_{h_{n}}\left\{\operatorname{grad}_{h_{n}}^{-} \phi_{j, k}\left(t_{n}\right)\right\} \longrightarrow \nabla \psi\left\{\begin{array}{l}
\text { strongly in } \mathbf{L}^{2}\left((0, a)^{2}\right) \\
\text { weakly in } \mathbf{W}_{2}^{1}\left((0, a)^{2}\right)
\end{array}\right.
$$

Therefore, (5.4) is obtained by setting $\mathbf{u}=\left(u^{r}, u^{z}\right)=\left(-\psi_{z} / r, \psi_{r} / r\right)$. Furthermore,

$$
\begin{aligned}
A_{h_{n}}\left\{\operatorname{grad}_{h_{n}}^{-} \phi_{j, k}\left(t_{n}\right)\right\} & \longrightarrow \nabla \psi \\
A_{h_{n}}\left\{(j+1) h_{n} \mathbf{v}_{j, k}^{\left(h_{n}\right)}\left(t_{n}\right)\right\} & \longrightarrow r \mathbf{u}
\end{aligned} \quad \text { strongly in } \mathbf{L}^{2}\left((0, a)^{2}\right),
$$

because (2.19) yields

$$
\begin{aligned}
& \iint_{0}^{a}\left|B_{h}\left\{\operatorname{grad}_{h}^{-} \phi_{j, k}\right\}-A_{h}\left\{\operatorname{grad}_{h}^{-} \phi_{j, k}\right\}\right|^{2} d r d z \\
& \quad \leq C h^{4} \sum_{j=0}^{N-1} \sum_{k=1}^{N}\left(\left|D_{h, r}^{+} \operatorname{grad}_{h}^{-} \phi_{j, k}\right|^{2}+\left|D_{h, z}^{+} \operatorname{grad}_{h}^{-} \phi_{j, k}\right|^{2}\right) \leq C^{\prime} h^{2}\left\|\zeta_{0}\right\|^{2} .
\end{aligned}
$$

It follows from (5.25) that

$$
A_{h_{n}}\left\{D_{h_{n}, s}^{+} \operatorname{grad}_{h_{n}}^{-} \phi_{j, k}\left(t_{n}\right)\right\} \longrightarrow \nabla \psi_{s} \quad \text { weakly in } \mathbf{L}^{2}\left((0, a)^{2}\right)
$$


where $s=r, z$. Indeed, this is obtained by using [6, Chapter VI, Lemma 3.1] for $(r, z) \in$ $(0, a-\epsilon) \times(0, a)$ with an arbitrary small constant $\epsilon>0$.

It is easy to see that

$$
A_{h}\left\{(j+1)^{\gamma} h^{\gamma}\right\}(r) \longrightarrow r^{\gamma} \begin{cases}\text { uniformly for } r \in[0, a] & \text { if } \gamma>0 \\ \text { uniformly for } r \in[\delta, a] & \text { if } \gamma<0\end{cases}
$$

as $h \rightarrow 0$, where $\delta>0$ is an arbitrary small constant, and $A_{h}\left\{(j+2)^{\gamma} h^{\gamma}\right\}$ converges to $r^{\gamma}$ in the same manner. To verify (5.29), we use

$$
\left|A_{h}\left\{(j+1)^{\gamma} h^{\gamma}\right\}-r^{\gamma}\right|=\left|\left(\left[\frac{r}{h}\right]+1\right)^{\gamma} h^{\gamma}-r^{\gamma}\right| \leq\left|(r+h)^{\gamma}-r^{\gamma}\right|
$$

The weak convergence of $A_{h_{n}}\left\{(j+1)^{1 / 2} h_{n}^{1 / 2} \zeta_{j, k}\left(t_{n}\right)\right\}$ (or a subsequence of it) in $L^{2}\left((0, a)^{2}\right)$ follows from (5.10). Let $f$ be an arbitrary function in $L^{2}\left((0, a)^{2}\right)$ which vanishes near $r=0$. Then, by (5.20), (5.28), and (5.29), we have

$$
\begin{aligned}
& \iint_{0}^{a} A_{h_{n}}\left\{(j+1)^{1 / 2} h_{n}^{1 / 2} \zeta_{j, k}\left(t_{n}\right)\right\} f d r d z \\
& \quad=-\iint_{0}^{a} \frac{A_{h_{n}}\left\{(j+2) h_{n} \Xi_{h_{n}} \phi_{j, k}\left(t_{n}\right)\right\}}{A_{h_{n}}\left\{(j+2) h_{n}\right\} A_{h_{n}}\left\{(j+1)^{3 / 2} h_{n}^{3 / 2}\right\}} f d r d z \\
& \quad \longrightarrow-\iint_{0}^{a} \frac{r \psi_{r r}-\psi_{r}+r \psi_{z z}}{r^{5 / 2}} f d r d z=\iint_{0}^{a} \frac{(\nabla \times \mathbf{u}) \cdot \mathbf{e}^{\theta}}{r^{1 / 2}} f d r d z .
\end{aligned}
$$

From this, (5.5) follows. Therefore, (5.10) brings (5.6). It is not hard to see that $\nabla \cdot \mathbf{u}=0$ in $(0, a)^{2}, \mathbf{u}(r, z)=\mathbf{u}(r, z+a), u^{r}(a, z)=0,\|\mathbf{u}\|<\infty$, and

$$
\left|\iint_{0}^{a} \mathbf{u} r d r d z\right|=\left|\iint_{0}^{a} \nabla \psi d r d z\right|=0 .
$$

These mean that $\mathbf{u} \in \tilde{\mathbf{X}}_{1}$. Noting that the equality $(d / d t)\left\langle\left\{\zeta_{j, k}, 1\right\}\right\rangle_{\Lambda_{h}}=0$ is derived from (4.8) and (5.9), we have $\left\langle\left\{\zeta_{j, k}, 1\right\}\right\rangle_{\Lambda_{h}}=\left\langle\left\{\zeta_{0, j, k}, 1\right\}\right\rangle_{\Lambda_{h}}$. It leads to (5.7) as $h=h_{n} \rightarrow 0$ and $t=t_{n} \rightarrow \infty$ because of (5.5) and (5.29).

Lastly, let us prove that $\mathbf{u}$ satisfies (5.1). By $\chi$, we denote an arbitrary function in $C^{2}([0, a] \times \mathbb{R})$ satisfying $\chi(0, z)=0, \chi(a, z)=$ const. $,\left|\lim _{r \rightarrow 0}\left(\chi_{r} / r\right)\right|<\infty, \lim _{r \rightarrow 0}\left(\chi_{z} / r\right)=0$, $\chi(r, z)=\chi(r, z+a)$, and $\left\|r^{-2} \Xi \chi\right\|<\infty$. Let $\left\{\chi_{j, k}\right\},\left\{g_{j, k}^{r}\right\}$ for $(j, k) \in\left(\Lambda_{h}^{r} \cup\{0\}\right) \times \mathbb{Z}$ and $\left\{g_{j, k}^{z}\right\}$ for $(j, k) \in \Lambda_{h}^{r} \times \mathbb{Z}$ be defined by

$$
\begin{gathered}
\chi_{j, k}=\chi\left(a(a-h)^{-1} j h, k h\right), \\
\mathbf{g}_{j, k}=\left(g_{j, k}^{r}, g_{j, k}^{z}\right)=\left(-\frac{1}{(j+1) h} D_{h, z}^{-} \chi_{j, k}, \frac{1}{(j+1) h} D_{h, r}^{-} \chi_{j, k}\right) .
\end{gathered}
$$


3338 Axisymmetric steady states of an inviscid incompressible fluid

Then, $\mathbf{g}_{j, k}$ satisfies $P_{\sigma, h} \mathbf{g}_{j, k}=\mathbf{g}_{j, k}$, that is, $\operatorname{div}_{h}^{-} \mathbf{g}_{j, k}=0, g_{0, k}^{r}=g_{N-1, k}^{r}=0$, and $\mathbf{g}_{j, k}=\mathbf{g}_{j, k+N}$. In addition, we have

$$
\sup _{\substack{h<r<a \\ 0<z<a}}\left|A_{h}\left\{(j+1)^{1 / 2} h^{1 / 2} \mathbf{g}_{j, k}\right\}-r^{1 / 2} \mathbf{g}\right| \leq C h^{1 / 2},
$$

where $\mathbf{g}=\left(-\chi_{z} / r, \chi_{r} / r\right)$. This is verified as

$$
\begin{aligned}
\left|A_{h}\left\{(j+1)^{1 / 2} h^{1 / 2} \mathbf{g}_{j, k}\right\}-r^{1 / 2} \mathbf{g}\right| \\
\quad=\left|\frac{\operatorname{grad}_{h}^{-} \chi_{[r / h],[z / h]}}{([r / h]+1)^{1 / 2} h^{1 / 2}}-\frac{\nabla \chi}{r^{1 / 2}}\right| \\
\quad \leq \frac{\left|\operatorname{grad}_{h}^{-} \chi_{[r / h],[z / h]}-\nabla \chi\right|}{([r / h]+1)^{1 / 2} h^{1 / 2}}+|\nabla \chi|\left|\frac{1}{([r / h]+1)^{1 / 2} h^{1 / 2}}-\frac{1}{r^{1 / 2}}\right| \\
\quad \leq \frac{\left|\operatorname{grad}_{h}^{-} \chi_{[r / h],[z / h]}-\nabla \chi\right|}{h^{1 / 2}}+|\nabla \chi|\left(\frac{1}{r^{1 / 2}}-\frac{1}{(r+h)^{1 / 2}}\right) \\
\quad \leq C h^{1 / 2} \sup _{\substack{0<r<a \\
0<z<a}}\left(\left|\nabla \chi_{r}\right|+\left|\nabla \chi_{z}\right|+\frac{|\nabla \chi|}{r}\right) .
\end{aligned}
$$

By (4.7), we have

$$
\begin{aligned}
-\left\langle\left\{\mathbf{V}_{j, k}, \mathbf{g}_{j, k}\right\}\right\rangle_{\Lambda_{h}}= & \frac{1}{2}\left\langle\left\{\left(\zeta_{j, k}+\zeta_{j+1, k}\right) D_{h, r}^{+} \phi_{j, k}, g_{j, k}^{r}\right\}\right\rangle_{\Lambda_{h}} \\
& +\frac{1}{2}\left\langle\left\{\left(\zeta_{j, k}+\zeta_{j, k+1}\right) D_{h, z}^{+} \phi_{j, k}, g_{j, k}^{z}\right\}\right\rangle_{\Lambda_{h}} \\
= & \left\langle\left\{\left(\zeta_{j, k} \mathbf{e}^{\theta}\right) \times\left((j+1) h \mathbf{v}_{j, k}^{(h)}\right), \mathbf{g}_{j, k}\right\}\right\rangle_{\Lambda_{h}}+F,
\end{aligned}
$$

where

$$
\begin{aligned}
F=\frac{1}{2}( & \left\langle\left\{\zeta_{j, k}\left(D_{h, r}^{+} \phi_{j, k}-D_{h, r}^{-} \phi_{j, k}\right), g_{j, k}^{r}\right\}\right\rangle_{\Lambda_{h}^{\prime}} \\
& +\left\langle\left\{\zeta_{j, k} D_{h, r}^{-} \phi_{j, k}, \frac{j}{j+1} g_{j-1, k}^{r}-g_{j, k}^{r}\right\}\right\rangle_{\Lambda_{h}} \\
& +\left\langle\left\{\zeta_{j, k}\left(D_{h, z}^{+} \phi_{j, k}-D_{h, z}^{-} \phi_{j, k}\right), g_{j, k}^{z}\right\}\right\rangle_{\Lambda_{h}} \\
& \left.+\left\langle\left\{\zeta_{j, k} D_{h, z}^{-} \phi_{j, k}, g_{j, k-1}^{z}-g_{j, k}^{z}\right\}\right\rangle_{\Lambda_{h}}\right),
\end{aligned}
$$

$\Lambda_{h}^{\prime}=\Lambda_{h}-\{(j, k) \mid j=N-1\}$. Using (5.10), (5.11), and (5.34) with $\left|r^{1 / 2} \mathbf{g}\right|<\infty$, we estimate $F$ as

$$
F \leq C\left(F_{1}+F_{2}+F_{3}\right)
$$


where

$$
\begin{aligned}
F_{1} & =\left(\int_{0}^{a} d z \int_{h}^{a-h}\left(A_{h}\left\{D_{h, r}^{-} \phi_{j, k}\right\}(r+h, z)-A_{h}\left\{D_{h, r}^{-} \phi_{j, k}\right\}(r, z)\right)^{2} d r\right)^{1 / 2}, \\
F_{2} & =\left(\int_{0}^{a} d z \int_{h}^{a}\left(A_{h}\left\{D_{h, z}^{-} \phi_{j, k}\right\}(r, z+h)-A_{h}\left\{D_{h, z}^{-} \phi_{j, k}\right\}(r, z)\right)^{2} d r\right)^{1 / 2}, \\
F_{3} & =\sup _{(j, k) \in \Lambda_{h}}(j+1)^{1 / 2} h^{1 / 2}\left|\frac{j}{j+1} g_{j-1, k}^{r}-g_{j, k}^{r}\right|+\sup _{(j, k) \in \Lambda_{h}}(j+1)^{1 / 2} h^{1 / 2}\left|g_{j, k-1}^{z}-g_{j, k}^{z}\right| \\
& \leq 2 h^{1 / 2} \sup _{(j, k) \in \Lambda_{h}}\left|D_{h, r}^{-} D_{h, z}^{-} \chi_{j, k}\right| \leq C h^{1 / 2} \sup _{\substack{0<r<a \\
0<z<a}}\left(\left|\nabla \chi_{r}\right|+\left|\nabla \chi_{z}\right|\right) .
\end{aligned}
$$

By (5.26) and the Lebesgue theorem, it follows that

$$
\begin{aligned}
F_{1} \leq & \left(\int_{0}^{a} d z \int_{h}^{a-h}\left(A_{h}\left\{D_{h, r}^{-} \phi_{j, k}\right\}(r+h, z)-\psi_{r}(r+h, z)\right)^{2} d r\right)^{1 / 2} \\
& +\left(\int_{0}^{a} d z \int_{h}^{a-h}\left(A_{h}\left\{D_{h, r}^{-} \phi_{j, k}\right\}(r, z)-\psi_{r}(r, z)\right)^{2} d r\right)^{1 / 2} \\
& +\left(\int_{0}^{a} d z \int_{h}^{a-h}\left(\psi_{r}(r+h, z)-\psi_{r}(r, z)\right)^{2} d r\right)^{1 / 2} \longrightarrow 0 \quad \text { as } h=h_{n} \longrightarrow 0, t=t_{n} \longrightarrow \infty
\end{aligned}
$$

In the same way, we deduce the convergence $F_{2} \rightarrow 0$, which implies that $F$ vanishes as $h=h_{n} \rightarrow 0$ and $t=t_{n} \rightarrow \infty$. Then, applying (5.5), (5.17) (with $\left.t_{n}=t_{n}^{\left(h_{n}\right)}\right),(5.26)$, and (5.34) to (5.36), we obtain $\langle(\nabla \times \mathbf{u}) \times \mathbf{u}, \mathbf{g}\rangle=0$. Since $\mathbf{g}$ is an arbitrary function in $\mathbf{X}_{1} \cap$ $\mathbf{C}^{1}((0, a] \times \mathbb{R})$, which is dense in $\mathbf{X}_{1},(5.1)$ is satisfied by $\mathbf{u}$.

Lemma 5.3. In Theorem 5.2, the generalized solution $\mathbf{u}$ belongs to $\mathbf{L}^{2}\left((0, a)^{2}\right)$, and

$$
\lim _{n \rightarrow \infty} \iint_{0}^{a}\left|A_{h_{n}}\left\{(j+1)^{\rho} h_{n}^{\rho} \mathbf{v}_{j, k}^{\left(h_{n}\right)}\left(t_{n}\right)\right\}-r^{\rho} \mathbf{u}\right|^{2} d r d z=0
$$

is valid for $\rho>0$.

Proof. From (5.12), we have

$$
\iint_{0}^{a}|\mathbf{u}|^{2} d r d z \leq \lim _{n \rightarrow \infty} \iint_{0}^{a}\left|A_{h_{n}}\left\{\mathbf{v}_{j, k}^{\left(h_{n}\right)}\left(t_{n}\right)\right\}\right|^{2} d r d z \leq C\left\|\zeta_{0}\right\|^{2} .
$$

The equality (5.41) with $\rho=1$ is no other than (5.26). Moreover, (5.41) with $\rho>1$ is easily verified by using (5.29) and

$$
\begin{aligned}
\left|A_{h}\left\{(j+1)^{\rho} h^{\rho} \mathbf{v}_{j, k}^{(h)}\right\}-r^{\rho} \mathbf{u}\right| \leq & \left|A_{h}\left\{(j+1)^{\rho-1} h^{\rho-1}\right\}\right|\left|A_{h}\left\{(j+1) h \mathbf{v}_{j, k}^{(h)}\right\}-r \mathbf{u}\right| \\
& +\left|A_{h}\left\{(j+1)^{\rho-1} h^{\rho-1}\right\}-r^{\rho-1}\right||r \mathbf{u}| .
\end{aligned}
$$


3340 Axisymmetric steady states of an inviscid incompressible fluid

Assume that $0<\rho<1$ and set

$$
\begin{gathered}
G(\rho)=\left(\iint_{0}^{a}\left|A_{h}\left\{(j+1)^{\rho} h^{\rho} \mathbf{v}_{j, k}^{(h)}\right\}-r^{\rho} \mathbf{u}\right|^{2} d r d z\right)^{1 / 2}, \\
G_{1}(\rho)=\left(\iint_{0}^{a}\left|A_{h}\left\{(j+1)^{\rho} h^{\rho} \mathbf{v}_{j, k}^{(h)}\right\}-A_{h}\left\{(j+1)^{\rho} h^{\rho}\right\} \mathbf{u}\right|^{2} d r d z\right)^{1 / 2}, \\
G_{2}(\rho)=\left(\iint_{0}^{a}\left|A_{h}\left\{(j+1)^{\rho} h^{\rho}\right\} \mathbf{u}-r^{\rho} \mathbf{u}\right|^{2} d r d z\right)^{1 / 2} .
\end{gathered}
$$

From

$$
\begin{aligned}
G_{1}(\rho) \leq & \left(\iint_{0}^{a}\left|A_{h}\left\{(j+1)^{2 \rho} h^{2 \rho}\right\}\left(A_{h}\left\{\mathbf{v}_{j, k}^{(h)}\right\}-\mathbf{u}\right)\right|^{2} d r d z\right)^{1 / 4} \\
& \cdot\left(\iint_{0}^{a}\left|A_{h}\left\{\mathbf{v}_{j, k}^{(h)}\right\}-\mathbf{u}\right|^{2} d r d z\right)^{1 / 4} \\
\leq & C\left(G_{1}(2 \rho)\right)^{1 / 2}
\end{aligned}
$$

we derive

$$
\begin{aligned}
G_{1}(\rho) & \leq C\left(G_{1}(2 \rho)\right)^{1 / 2} \leq C^{3 / 2}\left(G_{1}(4 \rho)\right)^{1 / 4} \\
& \leq \cdots \leq C^{\prime}\left(G_{1}\left(2^{v} \rho\right)\right)^{1 / 2^{\nu}} \leq C^{\prime}\left(G\left(2^{v} \rho\right)+G_{2}\left(2^{v} \rho\right)\right)^{1 / 2^{\nu}} .
\end{aligned}
$$

Here, $v$ is an integer such that $2^{v} \rho \geq 1$. Then,

$$
G(\rho) \leq G_{1}(\rho)+G_{2}(\rho) \leq C\left(G\left(2^{v} \rho\right)+G_{2}\left(2^{v} \rho\right)\right)^{1 / 2^{v}}+G_{2}(\rho) \longrightarrow 0
$$

as $h=h_{n} \rightarrow 0$ and $t=t_{n} \rightarrow \infty$. Hence, (5.41) is also valid for $\rho \in(0,1)$.

Remark 5.4. In the same way as in the proofs of Theorem 5.2 and Lemma 5.3, analogously to (5.41), we can prove the convergence for $\mathbf{v}_{0, j, k}^{(h)}\left(=\left.\mathbf{v}_{j, k}^{(h)}\right|_{t=0}\right)$ :

$$
\lim _{n \rightarrow \infty} \iint_{0}^{a}\left|A_{h_{n}}\left\{(j+1)^{\rho} h_{n}^{\rho} \mathbf{v}_{0, j, k}^{\left(h_{n}\right)}\right\}-r^{\rho} \mathbf{v}_{0}\right|^{2} d r d z=0
$$

for $\rho>0$, where $\mathbf{v}_{0}$ is a function in $\tilde{\mathbf{X}}_{1}$ such that $r^{-1}\left(\nabla \times \mathbf{v}_{0}\right) \cdot \mathbf{e}^{\theta}=\zeta_{0}$.

Lemma 5.5. Assume that $\mathbf{v}_{0} \in \tilde{\mathbf{X}}_{1}$ is not a generalized solution to (1.2), that is,

$$
\left\langle\left(\nabla \times \mathbf{v}_{0}\right) \times \mathbf{v}_{0}, \mathbf{f}\right\rangle \neq 0 \quad \text { for some } \mathbf{f} \in \mathbf{X}_{1} .
$$

Then, for $\mathbf{u}$ in Theorem 5.2 with $\zeta_{0}=r^{-1}\left(\nabla \times \mathbf{v}_{0}\right) \cdot \mathbf{e}^{\theta}$,

$$
\begin{gathered}
\left\|\mathbf{v}_{0}\right\|<\|\mathbf{u}\| \leq C \quad \text { if } \alpha<0, \\
\|\mathbf{u}\|<\left\|\mathbf{v}_{0}\right\| \quad \text { if } \alpha>0
\end{gathered}
$$


Proof. Note that (2.9) (with $f_{j, k}=D_{h, r}^{-} \phi_{j, k}, j_{1}=1, j_{2}=N$, and $\delta=1$ ) and (5.11) yield

$$
h^{2} \sum_{k=1}^{N} \frac{\left(D_{h, r}^{-} \phi_{N, k}\right)^{2}}{(N+1) h} \leq \frac{h^{2}}{a} \sum_{k=1}^{N}\left(h \sum_{j=1}^{N}\left(\left(D_{h, r}^{-} \phi_{j, k}\right)^{2}+\left(D_{h, r}^{-} D_{h, r}^{-} \phi_{j, k}\right)^{2}\right)\right) \leq C h\left\|\zeta_{0}\right\|^{2} .
$$

Then, since

$$
\left|\left\{\mathbf{v}_{j, k}^{(h)}\right\}\right|_{\bar{\Lambda}_{h}}^{2}=\iint_{0}^{a}\left|A_{h}\left\{(j+1)^{1 / 2} h^{1 / 2} \mathbf{v}_{j, k}^{(h)}\right\}\right|^{2} d r d z+h^{2} \sum_{k=1}^{N} \frac{\left(D_{h, r}^{-} \phi_{N, k}\right)^{2}}{(N+1) h}
$$

holds, we obtain

$$
\left|\left\{\mathbf{v}_{j, k}^{(h)}\right\}\right|_{\bar{\Lambda}_{h}}^{2} \longrightarrow\|\mathbf{u}\|^{2} \quad \text { as } h=h_{n} \longrightarrow 0, t=t_{n} \longrightarrow \infty
$$

by (5.41). Analogously, (5.48) yields $\left|\left\{\mathbf{v}_{0, j, k}^{(h)}\right\}\right|_{\bar{\Lambda}_{h}}^{2} \rightarrow\left\|\mathbf{v}_{0}\right\|^{2}$ as $h=h_{n} \rightarrow 0$. Therefore, from (5.15), we derive

$$
\begin{aligned}
& 0 \leq\|\mathbf{u}\|^{2}-\left\|\mathbf{v}_{0}\right\|^{2} \leq C\left\|\zeta_{0}\right\|^{2} \quad \text { if } \alpha<0 \text {, } \\
& \|\mathbf{u}\|^{2}-\left\|\mathbf{v}_{0}\right\|^{2} \leq 0 \quad \text { if } \alpha>0 .
\end{aligned}
$$

What remains is to prove $\|\mathbf{u}\| \neq\left\|\mathbf{v}_{0}\right\|$. Suppose that $\|\mathbf{u}\|=\left\|\mathbf{v}_{0}\right\|$ holds. Then (5.15) yields

$$
\int_{0}^{t}\left|\left\{\mathbf{V}_{j, k}\left(t^{\prime}\right)\right\}\right|_{\Lambda_{h}}^{2} d t^{\prime} \longrightarrow 0 \quad \text { as } h=h_{n} \longrightarrow 0, t=t_{n} \longrightarrow \infty
$$

It leads to

$$
\int_{0}^{T}\left|\left\{\mathbf{V}_{j, k}\left(t^{\prime}\right)\right\}\right|_{\Lambda_{h}}^{2} d t^{\prime} \longrightarrow 0 \quad \text { as } h=h_{n} \longrightarrow 0
$$

for any finite $T>0$. Applying this to (5.15), we have

$$
\left|\left\{\mathbf{v}_{j, k}^{(h)}(t)\right\}\right|_{\bar{\Lambda}_{h}}^{2}-\left|\left\{\mathbf{v}_{0, j, k}^{(h)}\right\}\right|_{\bar{\Lambda}_{h}}^{2} \longrightarrow 0 \quad \text { as } h=h_{n} \longrightarrow 0, \text { uniformly for } t \in[0, T] .
$$

Let $\theta$ be an arbitrary function in $C^{2}([0, a] \times \mathbb{R})$ such that $\theta(r, z)=\theta(r, z+a)$, and set $\theta_{j, k}=\theta(j h, k h)$. Then, analogously to $(5.14)$, by using (5.9), we obtain

$$
\begin{aligned}
\left|\left\langle\left\{\theta_{j, k}, \frac{d}{d t} \zeta_{j, k}\right\}\right\rangle_{\Lambda_{h}}\right| & =\left|\frac{\alpha}{2}\left\langle\left\{\theta_{j, k}, \mathbf{V}_{j, k} \cdot \operatorname{grad}_{h}^{+} \zeta_{j, k}+\left(\begin{array}{c}
\frac{j}{j+1} V_{j-1, k}^{r} \\
V_{j, k-1}^{z}
\end{array}\right) \cdot \operatorname{grad}_{h}^{-} \zeta_{j, k}\right\}\right\rangle_{\Lambda_{h}}\right| \\
& =\left|\frac{\alpha}{2}\left\langle\left\{\mathbf{V}_{j, k},\left(\begin{array}{c}
\left(\zeta_{j, k}+\zeta_{j+1, k}\right) D_{h, r}^{+} \theta_{j, k} \\
\left(\zeta_{j, k}+\zeta_{j, k+1}\right) D_{h, z}^{+} \theta_{j, k}
\end{array}\right)\right\}\right\rangle_{\Lambda_{h}}\right| \leq C\left|\left\{\mathbf{V}_{j, k}\right\}\right|_{\Lambda_{h}} .
\end{aligned}
$$


3342 Axisymmetric steady states of an inviscid incompressible fluid

For any $t \in[0, T]$, it follows that

$$
\begin{aligned}
\left|\left\langle\left\{\theta_{j, k}, \zeta_{j, k}(t)-\zeta_{0, j, k}\right\}\right\rangle_{\Lambda_{h}}\right| & \leq \int_{0}^{t}\left|\left\langle\left\{\theta_{j, k}, \frac{d}{d t} \zeta_{j, k}\right\}\right\rangle_{\Lambda_{h}}\right| d t \\
& \leq C \int_{0}^{T}\left|\left\{\mathbf{V}_{j, k}\left(t^{\prime}\right)\right\}\right|_{\Lambda_{h}} d t^{\prime} \\
& \leq C\left(T \int_{0}^{T}\left|\left\{\mathbf{V}_{j, k}\left(t^{\prime}\right)\right\}\right|_{\Lambda_{h}}^{2} d t^{\prime}\right)^{1 / 2} \\
& \longrightarrow 0 \text { as } h=h_{n} \longrightarrow 0 .
\end{aligned}
$$

This means that

$$
A_{h_{n}}\left\{(j+1)^{1 / 2} h_{n}^{1 / 2} \zeta_{j, k}(t)\right\} \longrightarrow r^{1 / 2} \zeta_{0} \quad \text { weakly in } L^{2}\left((0, a)^{2}\right) \text {, uniformly for } t \in[0, T] .
$$

It is easier than (5.48) to verify that $A_{h_{n}}\left\{(j+1)^{1 / 2} h_{n}^{1 / 2} \phi_{0, j, k}\right\}$ converges to $r^{1 / 2} \phi_{0}$ strongly in $L^{2}\left((0, a)^{2}\right)$, where $\phi_{0, j, k}=\left.\phi_{j, k}\right|_{t=0}$, and $\phi_{0}$ is a function such that $\mathbf{v}_{0}=\left(-\phi_{0 z} / r, \phi_{0 r} / r\right)$. Therefore, noting that

$$
\begin{aligned}
\left\langle\left\{\phi_{0, j, k}, \zeta_{j, k}\right\}\right\rangle_{\Lambda_{h}} & =-h^{2} \sum_{(j, k) \in \Lambda_{h}} \phi_{0, j, k} \frac{\Xi_{h} \phi_{j, k}}{(j+1) h} \\
& =-h^{2} \sum_{(j, k) \in \bar{\Lambda}_{h}} \phi_{0, j, k}\left(D_{h, r}^{+}\left(\frac{D_{h, r}^{-} \phi_{j, k}}{(j+1) h}\right)+\frac{D_{h, z}^{+} D_{h, z}^{-} \phi_{j, k}}{(j+1) h}\right) \\
& =\left\langle\left\{\mathbf{v}_{0, j, k}^{(h)}, \mathbf{v}_{j, k}^{(h)}\right\}\right\rangle_{\bar{\Lambda}_{h}},
\end{aligned}
$$

we have

$$
\left\langle\left\{\mathbf{v}_{0, j, k}^{(h)}, \mathbf{v}_{j, k}^{(h)}(t)\right\}\right\rangle_{\bar{\Lambda}_{h}}-\left|\left\{\mathbf{v}_{0, j, k}^{(h)}\right\}\right|_{\bar{\Lambda}_{h}}^{2}=\left\langle\left\{\phi_{0, j, k}, \zeta_{j, k}(t)-\zeta_{0, j, k}\right\}\right\rangle_{\Lambda_{h}} \longrightarrow 0
$$

as $h=h_{n} \rightarrow 0$, uniformly for $t \in[0, T]$. It leads to

$$
\left|\left\{\mathbf{v}_{j, k}^{(h)}(t)-\mathbf{v}_{0, j, k}^{(h)}\right\}\right|_{\bar{\Lambda}_{h}}^{2} \longrightarrow 0 \quad \text { as } h=h_{n} \longrightarrow 0, \text { uniformly for } t \in[0, T],
$$

because of (5.58). This implies that

$$
A_{h_{n}}\left\{(j+1)^{1 / 2} h_{n}^{1 / 2} \mathbf{v}_{j, k}^{\left(h_{n}\right)}(t)\right\} \longrightarrow r^{1 / 2} \mathbf{v}_{0}
$$

strongly in $\mathrm{L}^{2}\left((0, a)^{2}\right)$, uniformly for $t \in[0, T]$. Then it is clear from (5.29) that

$$
\begin{aligned}
& A_{h_{n}}\left\{(j+1) h_{n} \mathbf{v}_{j, k}^{\left(h_{n}\right)}(t)\right\} \longrightarrow r \mathbf{v}_{0} \\
& A_{h_{n}}\left\{\operatorname{grad}_{h_{n}}^{-} \phi_{j, k}(t)\right\} \longrightarrow \nabla \phi_{0}
\end{aligned} \quad \text { strongly in } \mathbf{L}^{2}\left((0, a)^{2}\right), \text { uniformly for } t \in[0, T]
$$

Integrating (5.36) over $(0, T)$ and letting $h=h_{n} \rightarrow 0$, we obtain $\left\langle\left(\nabla \times \mathbf{v}_{0}\right) \times \mathbf{v}_{0}, \mathbf{g}\right\rangle=0$ by the same procedure as in the proof of Theorem 5.2. It contradicts (5.49) and the equality $\|\mathbf{u}\|=\left\|\mathbf{v}_{0}\right\|$ does not hold. 
In (5.51), the nontriviality of $\mathbf{u}$, that is, the inequality $\|\mathbf{u}\|>0$ is guaranteed by (5.7) if $\iint_{0}^{a} \zeta_{0} r d r d z \neq 0$.

\section{Infinite number of solutions}

Let us consider the eigenvalue problem

$$
\begin{gathered}
\Xi \phi=-\mu r^{2} \phi, \\
\phi(0, z)=\phi(a, z)=0, \quad \phi(r, z)=\phi(r, z+a),
\end{gathered}
$$

with $\left|\lim _{r \rightarrow 0}\left(\phi_{r} / r\right)\right|<\infty$ and $\lim _{r \rightarrow 0}\left(\phi_{z} / r\right)=0$. Since

$$
\left\|r^{-1} \nabla \phi\right\|^{2}=-\left\langle\phi, r^{-2} \Xi \phi\right\rangle=\mu\|\phi\|^{2}
$$

is derived from (6.1), $\mu$ is real and positive if it exists.

Set $\phi=R^{(n)}(r) Z^{(n)}(z)$ with

$$
Z^{(n)}(z)= \begin{cases}\sin \frac{2 n \pi z}{a} & \text { for } n=1,2,3, \ldots, \\ \cos \frac{2 n \pi z}{a} & \text { for } n=0,-1,-2, \ldots\end{cases}
$$

Then $R^{(n)}$ is a solution to

$$
\begin{gathered}
L^{(n)} R^{(n)}=-\mu r^{2} R^{(n)}, \quad L^{(n)}=\frac{d^{2}}{d r^{2}}-\frac{1}{r} \frac{d}{d r}-\left(\frac{2 n \pi}{a}\right)^{2}, \\
R^{(n)}(0)=R^{(n)}(a)=0, \quad\left|\lim _{r \rightarrow 0} \frac{1}{r} \frac{d R^{(n)}}{d r}\right|<\infty .
\end{gathered}
$$

By setting $r=2^{1 / 2} \mu^{-1 / 4} s^{1 / 2}$ and $R^{(n)}(r)=S^{(n)}(s)$, we have

$$
\begin{gathered}
\frac{d^{2} S^{(n)}}{d s^{2}}+\left(1-\frac{2}{\mu^{1 / 2}}\left(\frac{n \pi}{a}\right)^{2} \frac{1}{s}\right) S^{(n)}=0, \\
S^{(n)}(0)=0, \quad\left|\lim _{s \rightarrow 0} \frac{d S^{(n)}}{d s}\right|<\infty \\
S^{(n)}\left(\frac{\mu^{1 / 2} a^{2}}{2}\right)=0
\end{gathered}
$$

According to [1, Chapter 14], (6.6) with (6.7) is satisfied by

$$
S^{(n)}(s)=\mathscr{F}_{0}\left(\mu^{-1 / 2}\left(\frac{n \pi}{a}\right)^{2}, s\right) .
$$

Here, $\mathscr{F}_{0}(\cdot, \cdot)$ denotes the regular Coulomb wave function of order zero, which is represented with Kummer's confluent hypergeometric function ${ }_{1} F_{1}(\cdot ; \cdot ; \cdot)$ as

$$
\mathscr{F}_{0}(\eta, s)=\left(\frac{2 \pi \eta}{e^{2 \pi \eta}-1}\right)^{1 / 2} s e^{-i s}{ }_{1} F_{1}(1-i \eta ; 2 ; 2 i s) \text {. }
$$


3344 Axisymmetric steady states of an inviscid incompressible fluid

In particular, $\mathscr{F}_{0}(0, s)=\sin s$. Because of $(6.8)$, the equality

$$
\mathscr{F}_{0}\left(\frac{(n \pi)^{2}}{2 \beta}, \beta\right)=0 \quad \text { for } \beta=\frac{\mu^{1 / 2} a^{2}}{2}
$$

should be satisfied. It is easy for $n=0$. If $n \neq 0$, then we deduce that

$$
\lim _{\beta \rightarrow 0} e^{-i \beta}{ }_{1} F_{1}\left(1-\frac{i(n \pi)^{2}}{2 \beta} ; 2 ; 2 i \beta\right)=(\text { a finite real number })>0
$$

from $[1,(14.6 .8)$ or $(14.6 .9)]$ and that

$$
\mathscr{F}_{0}\left(\frac{(n \pi)^{2}}{2 \beta}, \beta\right) \sim \sin \beta \quad \text { for } \beta \gg(n \pi)^{2}
$$

from $[1,(14.6 .5)]$. Therefore, there exists a set $\left\{\mu_{m, n}\right\}_{m \in \mathbb{N}}$ for each $n$ such that (6.11) with $\mu=\mu_{m, n}$ holds, $\left\{\mu_{m, n}\right\}$ does not have an accumulation point except $\infty$, and $\mu_{m, n} \sim$ $(2(m+l) \pi)^{2} / a^{4}$ with an integer $l$ as $m \rightarrow \infty$ (in particular, $\mu_{m, 0}=(2 m \pi)^{2} / a^{4}$ for every $m \in$ $\mathbb{N})$. Moreover, for an arbitrarily given $n$, each of $\left\{\mu_{m, n}\right\}$ is simple, because the irregular Coulomb wave function, which is used for a solution of (6.6) independent of (6.9), is excluded by (6.7). We arrange $\left\{\mu_{m, n}\right\}$ so that $\mu_{m, n}<\mu_{m+1, n}$.

Define $\left\{R^{(m, n)}\right\}_{m \in \mathbb{N}, n \in \mathbb{Z}}$ by

$$
R^{(m, n)}(r)=\mathscr{F}_{0}\left(\mu_{m, n}^{-1 / 2}\left(\frac{n \pi}{a}\right)^{2}, \frac{\mu_{m, n}^{1 / 2} r^{2}}{2}\right) .
$$

Then it is a smooth solution to (6.4) and (6.5) with $\mu=\mu_{m, n}$, and the orthogonality

$$
\int_{0}^{a} R^{(m, n)} R^{\left(m^{\prime}, n\right)} r d r=0 \quad \text { for } m \neq m^{\prime}
$$

follows from $\mu_{m, n} \neq \mu_{m^{\prime}, n}$ and

$$
\int_{0}^{a}\left(r^{-2} L^{(n)} R^{(m, n)}\right) R^{\left(m^{\prime}, n\right)} r d r=\int_{0}^{a} R^{(m, n)}\left(r^{-2} L^{(n)} R^{\left(m^{\prime}, n\right)}\right) r d r .
$$

It should be noted that $r^{-2} L^{(n)} R^{(n)}=0$ with (6.5) means that $R^{(n)} \equiv 0$ and the inverse of $r^{-2} L^{(n)}$ exists, because zero is not an eigenvalue. Therefore, by the theory on completely continuous operators, we derive that $\left\{R^{(m, n)}\right\}_{m \in \mathbb{N}}$ for any fixed $n \in \mathbb{Z}$ forms a complete orthogonal system in $L^{2}(0, a)$ with the weight function $r$. Let $\phi^{(m, n)}=R^{(m, n)}(r) Z^{(n)}(z)$. Then it is a smooth solution to (6.1) with $\mu=\mu_{m, n}$, and $\left\{\phi^{(m, n)}\right\}_{m \in \mathbb{N}, n \in \mathbb{Z}}$ is a complete orthogonal system in $L^{2}\left((0, a)^{2}\right)$ with the weight $r$.

The set $\left\{\mathbf{w}^{(m, n)}\right\}_{m \in \mathbb{N}, n \in \mathbb{Z}}$ defined by

$$
\mathbf{w}^{(m, n)}=\frac{\nabla \times\left(r^{-1} \phi^{(m, n)} \mathbf{e}^{\theta}\right)}{\left\|\nabla \times\left(r^{-1} \phi^{(m, n)} \mathbf{e}^{\theta}\right)\right\|}=\frac{\left(-\phi_{z}^{(m, n)} / r, \phi_{r}^{(m, n)} / r\right)}{\mu_{m, n}^{1 / 2}\left\|\phi^{(m, n)}\right\|}
$$


is proved to form a complete orthonormal (resp., orthogonal) system in $\widetilde{\mathbf{X}}_{0}$ (resp., $\tilde{\mathbf{X}}_{1}$ ) by using

$$
\nabla \times \nabla \times\left(r^{-1} \phi^{(m, n)} \mathbf{e}^{\theta}\right)=-\left(r^{-1} \Xi \phi^{(m, n)}\right) \mathbf{e}^{\theta}=\mu_{m, n} r \phi^{(m, n)} \mathbf{e}^{\theta} .
$$

It corresponds to $\left\{\mathbf{w}^{(k)}\right\}_{k \in \mathbb{N}}$ : the set of basis functions in the Galerkin method used in [9]. As was mentioned in Section 1, const. $\mathbf{w}^{(m, n)}$ for any $m$ and $n$ is an exact solution to (1.2). Indeed,

$$
\left(\nabla \times \mathbf{w}^{(m, n)}\right) \times \mathbf{w}^{(m, n)}=\frac{1}{2} \nabla\left(\frac{\phi^{(m, n)}}{\left\|\phi^{(m, n)}\right\|}\right)^{2} .
$$

Since $\phi^{(m, 0)}=R^{(m, 0)}=\sin \left(m \pi r^{2} / a^{2}\right)$, we have

$$
\mathbf{w}^{(m, 0)}=\left(\frac{2}{a^{3 / 2}} \cos \frac{m \pi r^{2}}{a^{2}}\right) \mathbf{e}^{z} .
$$

This leads to

$$
\iint_{0}^{a}\left(\nabla \times \mathbf{w}^{(m, 0)}\right) \cdot \mathbf{e}^{\theta} d r d z=\frac{2}{a^{1 / 2}}\left(1-(-1)^{m}\right),
$$

while

$$
\iint_{0}^{a}\left(\nabla \times \mathbf{w}^{(m, n)}\right) \cdot \mathbf{e}^{\theta} d r d z=0 \quad \text { for } n \neq 0
$$

follows from the definition of $Z^{(n)}$.

Noting (5.7), that is, the conservation of circulation around the square domain $(0, a)^{2}$, we restrict $\widetilde{\mathbf{X}}_{1}$ to a subspace of itself:

$$
\mathbf{Y}=\left\{\mathbf{f} \in \tilde{\mathbf{X}}_{1} \mid \iint_{0}^{a}(\nabla \times \mathbf{f}) \cdot \mathbf{e}^{\theta} d r d z=\frac{4}{a^{1 / 2}}\right\}
$$

(the value $4 a^{-1 / 2}$ is only for convenience). Then, $\mathbf{E}=\left\{\mathbf{w}^{(2 j-1,0)}\right\}_{j \in \mathbb{N}}$ is a subset of $\mathbf{Y}$. It is clear that $c \mathbf{w}^{(m, n)}$ is not an element of $\mathbf{Y}-\mathbf{E}$ whatever $m \in \mathbb{N}, n \in \mathbb{Z}$, and $c \in \mathbb{R}$ are.

Theorem 6.1. By Theorem 5.2, the system (4.8) with $\alpha<0$ generates a set $\left\{\mathbf{u}_{k}\right\}_{k \in \mathbb{N}}$ such that each $\mathbf{u}_{k}$ is an axisymmetric generalized solution to (1.2) belonging to $\mathbf{Y}-\mathbf{E}$ and

$$
1<\left\|\mathbf{u}_{1}\right\|<\left\|\mathbf{u}_{2}\right\|<\left\|\mathbf{u}_{3}\right\|<\ldots
$$

Proof. Let $\mathbf{v}_{0}=\delta_{1} \mathbf{w}^{(1,0)}+\left(1-\delta_{1}\right) \mathbf{w}^{(3,0)}$ with a constant $\delta_{1} \in(-\infty, 0) \cup(1, \infty)$. Then, $\mathbf{v}_{0} \in$ $\mathbf{Y}-\mathbf{E}$ and $\left\|\mathbf{v}_{0}\right\|^{2}=\delta_{1}^{2}+\left(1-\delta_{1}\right)^{2}>1$. By Theorem 5.2 with $\alpha<0$ and $\zeta_{0}=r^{-1}\left(\nabla \times \mathbf{v}_{0}\right)$. $\mathbf{e}^{\theta}$, an axisymmetric generalized solution $\mathbf{u}=\mathbf{u}_{1} \in \mathbf{Y}$ to (1.2) is generated. Clearly, $\mathbf{v}_{0}$ is not a solution to (1.2), that is, (5.49) is valid. Therefore, $\left\|\mathbf{u}_{1}\right\|>1$ follows from (5.50). It means that $\mathbf{u}_{1} \notin \mathbf{E}$, because $\|\mathbf{w}\|=1$ for any $\mathbf{w} \in \mathbf{E}$. Redefine $\mathbf{v}_{0}$ by $\mathbf{v}_{0}=\delta_{2} \mathbf{w}^{(1,0)}+(1-$ $\left.\delta_{2}\right) \mathbf{w}^{(3,0)}$, where $\delta_{2} \in(-\infty, 0) \cup(1, \infty)$ is a constant satisfying $\left(\left\|\mathbf{v}_{0}\right\|^{2}=\right) \delta_{2}^{2}+\left(1-\delta_{2}\right)^{2} \geq$ $\left\|\mathbf{u}_{1}\right\|^{2}$. Then, in the same way as $\mathbf{u}_{1}$, we obtain a new generalized solution $\mathbf{u}_{2} \in \mathbf{Y}-\mathbf{E}$ which satisfies $\left\|\mathbf{u}_{2}\right\|>\left\|\mathbf{u}_{1}\right\|$. Repeating this process, we complete the proof. 
3346 Axisymmetric steady states of an inviscid incompressible fluid

\section{Acknowledgments}

The author thanks Professor Shun Shimomura for his useful suggestion on (6.6). This work was partly supported by Grant-in-Aid for Scientific Research no. 15740114 from the Japan Ministry of Education, Culture, Sports, Science and Technology.

\section{References}

[1] M. Abramowitz and I. A. Stegun, Handbook of Mathematical Functions, with Formulas, Graphs, and Mathematical Tables, National Bureau of Standards Applied Mathematics Series, vol. 55, U.S. Government Printing Office, Washington, DC, 1964.

[2] A. Ambrosetti and M. Struwe, Existence of steady vortex rings in an ideal fluid, Arch. Rational Mech. Anal. 108 (1989), no. 2, 97-109.

[3] V. I. Arnold and B. A. Khesin, Topological Methods in Hydrodynamics, Applied Mathematical Sciences, vol. 125, Springer, New York, 1998.

[4] L. E. Fraenkel and M. S. Berger, A global theory of steady vortex rings in an ideal fluid, Acta Math. 132 (1974), 13-51.

[5] A. Friedman and B. Turkington, Vortex rings: existence and asymptotic estimates, Trans. Amer. Math. Soc. 268 (1981), no. 1, 1-37.

[6] O. A. Ladyzhenskaya, The Boundary Value Problems of Mathematical Physics, Applied Mathematical Sciences, vol. 49, Springer, New York, 1985.

[7] H. K. Moffatt, Structure and stability of solutions of the Euler equations: a Lagrangian approach, Philos. Trans. Roy. Soc. London Ser. A 333 (1990), no. 1631, 321-342.

[8] W.-M. Ni, On the existence of global vortex rings, J. Analyse Math. 37 (1980), 208-247.

[9] T. Nishiyama, Pseudo-advection method for the axisymmetric stationary Euler equations, ZAMM Z. Angew. Math. Mech. 81 (2001), no. 10, 711-715.

[10] Magnetohydrodynamic approach to solvability of the three-dimensional stationary Euler equations, Glasg. Math. J. 44 (2002), no. 3, 411-418.

[11] Construction of solutions to the two-dimensional stationary Euler equations by the pseudo-advection method, Arch. Math. (Basel) 81 (2003), no. 4, 467-477.

[12] Construction of three-dimensional stationary Euler flows from pseudo-advected vorticity equations, R. Soc. Lond. Proc. Ser. A Math. Phys. Eng. Sci. 459 (2003), no. 2038, 2393-2398.

[13] G. K. Vallis, G. F. Carnevale, and W. R. Young, Extremal energy properties and construction of stable solutions of the Euler equations, J. Fluid Mech. 207 (1989), 133-152.

Takahiro Nishiyama: Department of Intelligent Mechanical Engineering, Faculty of Engineering, Fukuoka Institute of Technology, Fukuoka 8110295, Japan

Current address: Department of Applied Science, Faculty of Engineering, Yamaguchi University, Ube 7558611, Japan

E-mail address: t-nishi@yamaguchi-u.ac.jp 


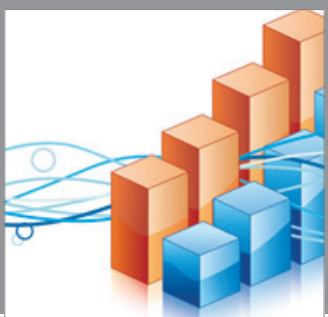

Advances in

Operations Research

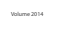

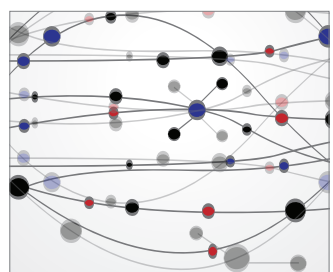

\section{The Scientific} World Journal
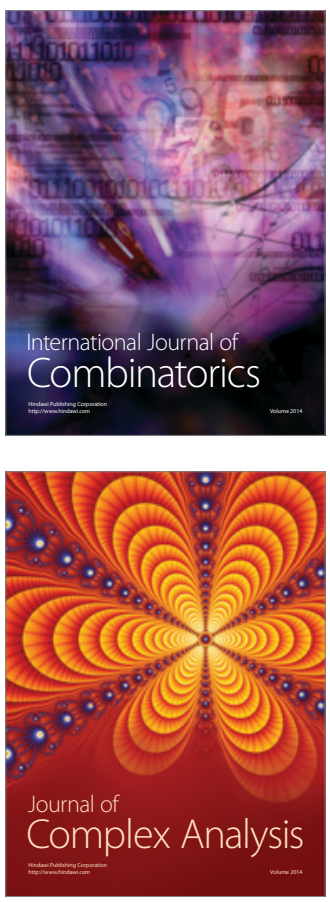

International Journal of

Mathematics and

Mathematical

Sciences
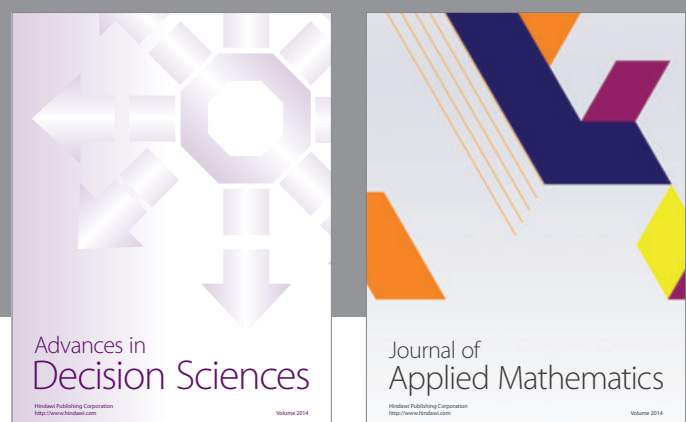

Journal of

Applied Mathematics
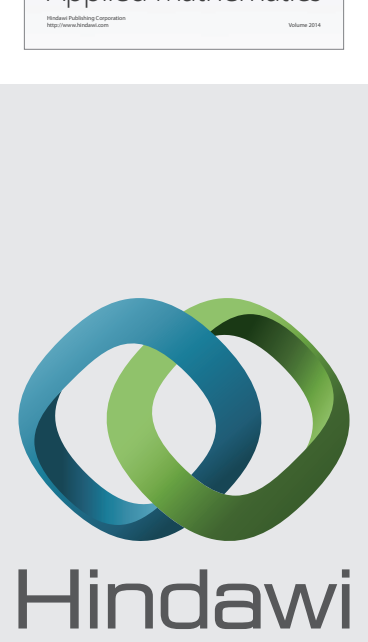

Submit your manuscripts at http://www.hindawi.com
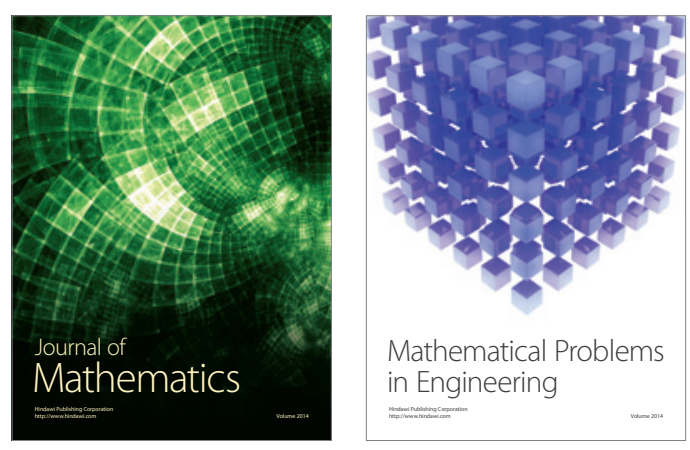

Mathematical Problems in Engineering
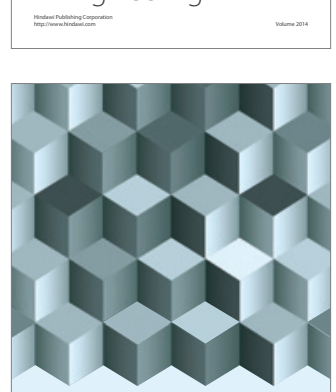

Journal of

Function Spaces
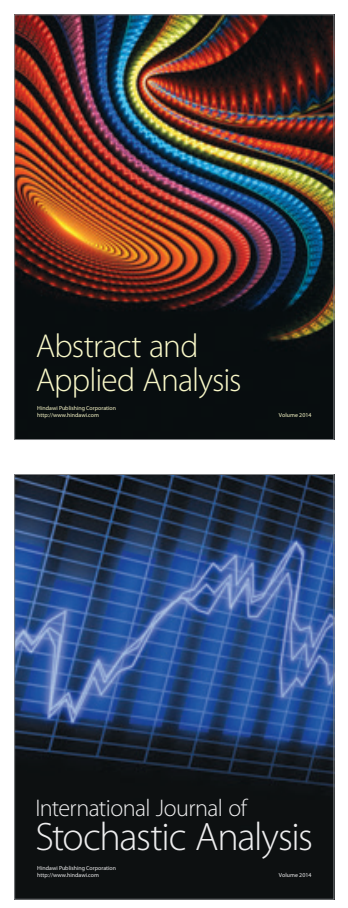

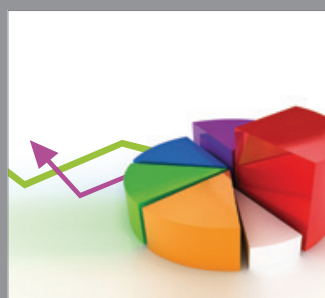

ournal of

Probability and Statistics

Promensencen
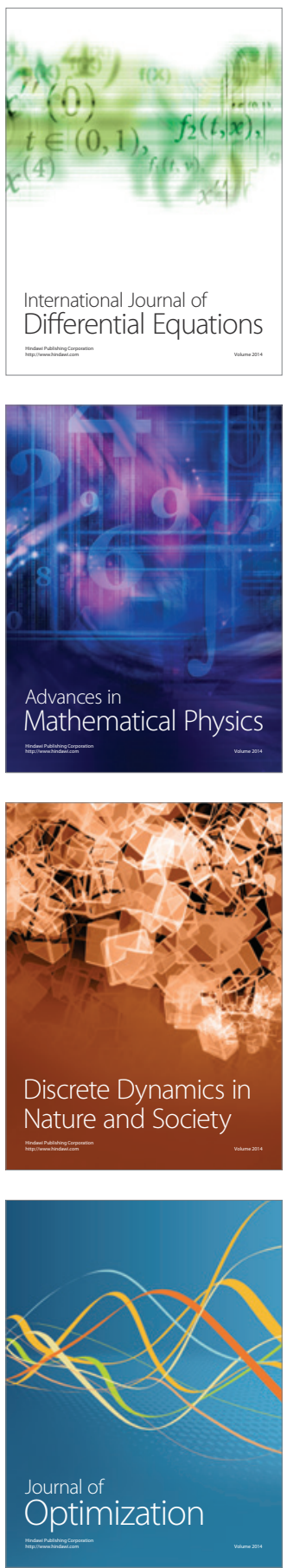